Draft Version November 13, 2018

Preprint typeset using $\mathrm{LATE}_{\mathrm{E}} \mathrm{X}$ style emulateapj v. 03/07/07

\title{
IMPROVED SPECTROSCOPIC PARAMETERS FOR TRANSITING PLANET HOSTS
}

\author{
Guillermo Torres ${ }^{1}$, Debra A. Fischer ${ }^{2}$, Alessandro Sozzetti ${ }^{3}$, Lars A. Buchhave ${ }^{4}$, Joshua N. Winn ${ }^{5}$, Matthew \\ J. Holman ${ }^{1}$, and Joshua A. Carter ${ }^{1}$ \\ Draft version November 13, 2018
}

\begin{abstract}
We report homogeneous spectroscopic determinations of the effective temperature, metallicity, and projected rotational velocity for the host stars of 56 transiting planets. Our analysis is based primarily on the Stellar Parameter Classification (SPC) technique. We investigate systematic errors by examining subsets of the data with two other methods that have often been used in previous studies (SME and MOOG). The SPC and SME results, both based on comparisons between synthetic spectra and actual spectra, show strong correlations between $T_{\mathrm{eff}},[\mathrm{Fe} / \mathrm{H}]$, and $\log g$ when solving for all three quantities simultaneously. In contrast the MOOG results, based on a more traditional curve-of-growth approach, show no such correlations. To combat the correlations and improve the accuracy of the temperatures and metallicities, we repeat the SPC analysis with a constraint on $\log g$ based on the mean stellar density that can be derived from the analysis of the transit light curves. Previous studies that have not taken advantage of this constraint have been subject to systematic errors in the stellar masses and radii of up to $20 \%$ and $10 \%$, respectively, which can be larger than other observational uncertainties, and which also cause systematic errors in the planetary mass and radius.

Subject headings: planetary systems — stars: abundances — stars: fundamental parameters techniques: spectroscopic
\end{abstract}

\section{INTRODUCTION}

In recent years the number of extrasolar transiting planets has expanded considerably, with discoveries being made at a rapid pace both from the ground and increasingly also from space by the CoRoT and Kepler missions. With this large assembly of data, studies have begun to focus on examining patterns and correlations among the global properties of these planets and their parent stars, and what this can tell us about planet formation and evolution.

While the characteristics of some of these systems are very well known (e.g., HD 209458, HD 189733, TrES-1), those of others are much less well determined and have remained so since their discovery. Our knowledge of the planetary properties depends critically on understanding the parent stars. This is because, for transiting systems, the light curves only give information on the size of the planet relative to that of the star $\left(R_{p} \propto R_{\star}\right)$, and spectroscopic observations only reveal the mass of the planet if we know the mass of the $\operatorname{star}\left(M_{p} \propto M_{\star}^{2 / 3}\right)$. The stellar mass and radius, in turn, depend on other properties that can be gleaned from the stars' spectra such as the effective temperature $\left(T_{\text {eff }}\right)$, surface gravity $(\log g)$, and chemical composition (commonly represented by $[\mathrm{Fe} / \mathrm{H}]$ ).

For many of the known transiting planet systems, follow-up photometric observations have been undertaken after the initial discovery of the planet, usually

\footnotetext{
${ }^{1}$ Harvard-Smithsonian Center for Astrophysics, 60 Garden St., Cambridge, MA 02138, USA; e-mail: gtorres@cfa.harvard.edu

2 Dept. of Astronomy, Yale University, New Haven, CT 06511, USA

${ }^{3}$ INAF - Osservatorio Astronomico di Torino, 10025 Pino Torinese, Italy

${ }^{4}$ Niels Bohr Institute, Copenhagen University, Denmark

${ }^{5}$ Dept. of Physics, and Kavli Institute for Astrophysics and Space Research, Massachusetts Institute of Technology, Cambridge, MA 02139, USA
}

for the purpose of measuring the times of mid-transit and seeking departures from strict periodicity (transit timing variations) that may indicate the presence of additional bodies in the system. These new transit light curve observations have also served to improve the radius determinations in many cases. However, it is much less common for known transiting systems to be re-observed spectroscopically. As a result, our knowledge of the stellar properties is often limited by whatever information was reported in the discovery papers, which is sometimes preliminary. Inaccuracies in the stellar $T_{\text {eff }}, \log g$, and $[\mathrm{Fe} / \mathrm{H}]$ propagate through to the determination of the planetary properties, and may obscure correlations with other quantities and prevent us from gaining valuable insight into the nature of planets. To make matters worse, the methods of determining $T_{\text {eff }}, \log g$, and $[\mathrm{Fe} / \mathrm{H}]$ in the literature are highly inhomogeneous, as they have been carried out by many groups using different assumptions and methodologies.

In one of the few studies to redetermine spectroscopic properties for transiting planet hosts in a uniform way, Ammler-von Eiff et al. (2009) obtained new temperatures, surface gravities, and metallicities for 13 systems based on new or existing spectra. They combined their determinations with those for 11 additional systems made by others using similar techniques, and compiled a list of 24 host stars with uniformly derived properties. Comparison with results for the same stars by other groups uncovered significant systematic differences in some cases, the causes of which are unknown.

One of the motivations for the present paper is to derive spectroscopic properties in a homogeneous manner for a much larger sample of more than 50 transiting planet hosts, and thereby reduce any dispersion in the stellar and planetary properties that is caused by the variety of methodologies used in the past. For this we make use of the Stellar Parameter Classification (SPC) 
technique introduced by Buchhave et al. (2012). We also wish to understand potential systematic errors in such determinations. In particular, it has been recognized for some time (e.g., Sozzetti et al. 2007; Holman et al. 2007; Winn et al. 2008) that surface gravities are often poorly constrained by spectral analyses. This is an unfortunate limitation, because the surface gravity would otherwise help to establish the luminosity of the star (and hence its radius) by placing it on the H-R diagram.

In cases for which the surface gravity is poorly constrained, the use of an external constraint on the luminosity becomes very important to allow accurate determinations of the mass and radius of the star. However, a detail that has usually been overlooked is that the determinations of other spectroscopic quantities such as the temperature and metallicity can also be affected by the poor constraint on gravity. This is because the uncertainties in $T_{\text {eff }}$ and $[\mathrm{Fe} / \mathrm{H}]$ are strongly correlated with $\log g$ in at least some of the commonly used analysis techniques. This can be a significant source of systematic error. Therefore, a second goal of our work is to compare spectroscopic determinations for a subset of the sample obtained using two additional procedures widely employed in previous studies, and to investigate and quantify systematics errors stemming from the weakly constrained gravities.

Ultimately the stellar quantities that enter into the calculation of the planetary characteristics are the masses and radii inferred from $T_{\text {eff }}, \log g$, and $[\mathrm{Fe} / \mathrm{H}]$, as mentioned earlier. A third objective of the present paper is to study and quantify how errors in the spectroscopic properties propagate into the stellar masses and radii.

The paper is organized as follows. In Sect. 2 we report our spectroscopic observations, which consist of new and archival spectra obtained with three different telescopes. Our spectroscopic analysis techniques are described in Sect. 3. Our results with and without the application of external constraints on the surface gravity are presented in Sect. 4. Also presented there are the final results from this work. These are then compared with work by others in Sect. 5. In Sect. 6] we study the impact of the different assumptions regarding $\log g$ on the stellar masses and radii. We discuss our results in Sect. 7, and end with a summary of our conclusions in Sect. 8 .

\section{SPECTROSCOPIC OBSERVATIONS AND REDUCTIONS}

For this work we have relied in part on new spectra collected with the TRES instrument (Fürész 2008) on the $1.5 \mathrm{~m}$ Tillinghast reflector at the F. L. Whipple Observatory (Mount Hopkins, AZ), and also on publicly available spectra obtained with the HIRES instrument (Vogt et al. 1994) on the Keck I telescope (Mauna Kea, HI) and with the FIES instrument (Djupvic \& Andersen 2010) on the $2.5 \mathrm{~m}$ Nordic Optical Telescope (La Palma, Spain). The archival spectra have generally not been used previously for a determination of the stellar properties, and even when they have, we have redetermined those properties here using different methodologies for comparison purposes.

We obtained new TRES spectra for 22 stars (see Table 11). They cover the wavelength range $\sim 3860-9100 \AA$ and were taken at a typical resolving power of $R \approx 48,000$ with the medium fiber of that instrument. The signal-
TABLE 1

EXOPLANET HOST STARS WITH NEW TRES SPECTRA.

\begin{tabular}{|c|c|c|c|c|c|c|c|c|}
\hline \multirow[b]{2}{*}{ Star } & \multirow[b]{2}{*}{$N_{\text {obs }}$} & \multirow[b]{2}{*}{ SNR } & \multicolumn{2}{|c|}{$\begin{array}{c}\text { SPC } \\
\text { analysis }\end{array}$} & \multicolumn{2}{|c|}{$\begin{array}{c}\text { SME } \\
\text { analysis }\end{array}$} & \multicolumn{2}{|c|}{$\begin{array}{l}\text { MOOG } \\
\text { analysis }\end{array}$} \\
\hline & & & $\mathrm{U}$ & $\mathrm{C}$ & $\mathrm{U}$ & $\mathrm{C}$ & $\mathrm{U}$ & $\mathrm{C}$ \\
\hline HАТ-P-3 & 3 & 36 & $\mathrm{X}$ & $\mathrm{X}$ & - & - & - & - \\
\hline HAT-P-4 & 1 & 51 & $\mathrm{X}$ & $\mathrm{X}$ & - & - & - & - \\
\hline НАТ-P-5 & 1 & 44 & $\mathrm{X}$ & $\mathrm{X}$ & - & - & $\mathrm{X}$ & $\mathrm{X}$ \\
\hline HAT-P-7 & 1 & 68 & $\mathrm{X}$ & $\mathrm{X}$ & - & - & - & - \\
\hline HAT-P-9 & 4 & 42 & $\mathrm{X}$ & $\mathrm{X}$ & - & - & $\mathrm{X}$ & $\mathrm{X}$ \\
\hline HAT-P-10 & 1 & 27 & $\mathrm{X}$ & $\mathrm{X}$ & - & - & - & - \\
\hline HAT-P-13 & 1 & 38 & $\mathrm{X}$ & $\mathrm{X}$ & - & - & - & - \\
\hline HAT-P-15 & 1 & 42 & $\mathrm{X}$ & $\mathrm{X}$ & - & - & - & - \\
\hline НАТ-P-17 & 1 & 56 & $\mathrm{X}$ & $\mathrm{X}$ & - & - & - & - \\
\hline HAT-P-19 & 1 & 37 & $\mathrm{X}$ & $\mathrm{X}$ & - & - & - & - \\
\hline HAT-P-20 & 1 & 30 & $\mathrm{X}$ & $\mathrm{X}$ & - & - & - & - \\
\hline HAT-P-21 & 1 & 37 & $\mathrm{X}$ & $\mathrm{X}$ & - & - & - & - \\
\hline HAT-P-22 & 1 & 81 & $\mathrm{X}$ & $\mathrm{X}$ & - & - & - & - \\
\hline HAT-P-24 & 1 & 57 & $\mathrm{X}$ & $\mathrm{X}$ & - & - & - & - \\
\hline HAT-P-26 & 1 & 51 & $\mathrm{X}$ & $\mathrm{X}$ & - & - & - & - \\
\hline HAT-P-29 & 1 & 48 & $\mathrm{X}$ & $\mathrm{X}$ & - & - & - & - \\
\hline HD 147506 & 2 & 98 & $\mathrm{X}$ & $\mathrm{X}$ & - & - & - & - \\
\hline WASP-2 & 1 & 44 & $\mathrm{X}$ & $\mathrm{X}$ & - & - & $\mathrm{X}$ & $\mathrm{X}$ \\
\hline WASP-3 & 1 & 62 & $\mathrm{X}$ & $\mathrm{X}$ & - & - & $\mathrm{X}$ & $\mathrm{X}$ \\
\hline WASP-10 & 2 & 35 & $\mathrm{X}$ & $\mathrm{X}$ & - & - & $\mathrm{X}$ & $\mathrm{X}$ \\
\hline WASP-13 & 3 & 78 & $\mathrm{X}$ & $\mathrm{X}$ & - & - & $\mathrm{X}$ & $\mathrm{X}$ \\
\hline WASP-14 & 4 & 104 & $\mathrm{X}$ & $\mathrm{X}$ & - & - & $\mathrm{X}$ & $\mathrm{X}$ \\
\hline
\end{tabular}

Note. - $\mathrm{U}=$ unconstrained analysis ( $\log g$ free); $\mathrm{C}=$ constrained analysis ( $\log g$ fixed to best value from photometry); $\mathrm{X}$ indicates the method has been applied to this star.

to-noise ratios (SNRs) for individual exposures range between 27 and 130 per resolution element of $6.2 \mathrm{~km} \mathrm{~s}^{-1}$, and refer to the region of the $\mathrm{Mg}$ I b triplet $(\sim 5200 \AA)$. For stars with multiple exposures the SNR reported is the average. All spectra were reduced using the procedures described by Buchhave et al. (2010a, b).

The archival HIRES spectra (Table 2) were obtained with the typical setup employed for extrasolar planet searches with that instrument. These spectra cover the full optical range and were gathered at two different nominal resolving powers of $R \approx 68,000$ and $R \approx 51,000$. The SNRs in the $\mathrm{Mgb}$ region range from about 40 to 360 per resolution element $\left(4.4 \mathrm{~km} \mathrm{~s}^{-1}\right.$ and $5.9 \mathrm{~km} \mathrm{~s}^{-1}$, respectively). Many of the spectra available from this instrument used an iodine cell in the beam to impose a dense set of molecular absorption lines that serve as a fiducial in determining precise Doppler shifts. For the present work we analyzed only the spectra taken without the iodine cell (referred to as "templates"). They were reduced following standard procedures for this instrument.

The archival FIES spectra cover the wavelength range from about $3600 \AA$ to $7400 \AA$, and were obtained with the medium fiber of that spectrograph at a typical resolving power of $R \approx 46,000$. The SNRs at $\sim 5200 \AA$ for individual exposures range from 21 to 220 per resolution element of $6.5 \mathrm{~km} \mathrm{~s}^{-1}$. The list of stars is given in Table 3. Reductions were carried out as described by Buchhave et al. (2010a, b) using nightly flatfield, bias, and dark frames, as well as thorium-argon exposures taken immediately before and after the science exposure. This instrument does not use an iodine cell, and relies instead on its intrinsic stability to enable the measurement of precise radial velocities of exoplanet hosts. As a result, there are usually many more spectra of each object available for 
TABLE 2

EXOPLANET HOST STARS WITH ARCHIVAL HIRES SPECTRA.

\begin{tabular}{|c|c|c|c|c|c|c|c|c|}
\hline \multirow[b]{2}{*}{ Star } & \multirow[b]{2}{*}{$N_{\text {obs }}$} & \multirow[b]{2}{*}{ SNR } & \multicolumn{2}{|c|}{$\begin{array}{c}\text { SPC } \\
\text { analysis } \\
\end{array}$} & \multicolumn{2}{|c|}{$\begin{array}{c}\text { SME } \\
\text { analysis }\end{array}$} & \multicolumn{2}{|c|}{$\begin{array}{l}\text { MOOG } \\
\text { analysis }\end{array}$} \\
\hline & & & $\mathrm{U}$ & C & $\mathrm{U}$ & $\mathrm{C}$ & $\mathrm{U}$ & $\mathrm{C}$ \\
\hline CoRoT-1 & 1 & 84 & $\mathrm{X}$ & $\mathrm{X}$ & $\mathrm{X}$ & $\mathrm{X}$ & $\mathrm{X}$ & $\mathrm{X}$ \\
\hline CoRoT-2 & 1 & 128 & $\mathrm{X}$ & $\mathrm{X}$ & $\mathrm{X}$ & $\mathrm{X}$ & $\mathrm{X}$ & $\mathrm{X}$ \\
\hline CoRoT-7 & 1 & 103 & - & $\mathrm{X}$ & $\mathrm{X}$ & $\mathrm{X}$ & $\mathrm{X}$ & $\mathrm{X}$ \\
\hline HAT-P-3 & 1 & 163 & $\mathrm{X}$ & $\mathrm{X}$ & $\mathrm{L}$ & - & - & - \\
\hline НАТ-P-4 & 1 & 202 & $\mathrm{X}$ & $\mathrm{X}$ & $\mathrm{L}$ & $\mathrm{L}$ & - & - \\
\hline HAT-P-6 & 1 & 303 & $\mathrm{X}$ & $\mathrm{X}$ & $\mathrm{X}$ & $\mathrm{X}$ & $\mathrm{X}$ & $\mathrm{X}$ \\
\hline HAT-P-7 & 1 & 344 & $\mathrm{X}$ & $\mathrm{X}$ & $\mathrm{L}$ & - & - & - \\
\hline HAT-P-8 & 1 & 276 & $\mathrm{X}$ & $\mathrm{X}$ & $\mathrm{L}$ & $\mathrm{L}$ & - & - \\
\hline HAT-P-10 & 1 & 179 & $\mathrm{X}$ & $\mathrm{X}$ & $\mathrm{L}$ & - & - & - \\
\hline HAT-P-11 & 3 & 319 & $\mathrm{X}$ & $\mathrm{X}$ & $\mathrm{L}$ & $\mathrm{L}$ & - & - \\
\hline HAT-P-13 & 3 & 192 & $\mathrm{X}$ & $\mathrm{X}$ & $\mathrm{L}$ & $\mathrm{L}$ & - & - \\
\hline НАТ-P-14 & 1 & 284 & $\mathrm{X}$ & $\mathrm{X}$ & $\mathrm{L}$ & $\mathrm{L}$ & - & - \\
\hline HAT-P-15 & 1 & 148 & $\mathrm{X}$ & $\mathrm{X}$ & $\mathrm{L}$ & $\mathrm{L}$ & - & - \\
\hline HAT-P-16 & 1 & 206 & $\mathrm{X}$ & $\mathrm{X}$ & $\mathrm{L}$ & $\mathrm{L}$ & - & - \\
\hline HAT-P-17 & 1 & 247 & $\mathrm{X}$ & $\mathrm{X}$ & $\mathrm{L}$ & $\mathrm{L}$ & - & - \\
\hline HAT-P-18 & 1 & 125 & $\mathrm{X}$ & $\mathrm{X}$ & $\mathrm{L}$ & $\mathrm{L}$ & - & - \\
\hline HAT-P-19 & 1 & 104 & $\mathrm{X}$ & $\mathrm{X}$ & $\mathrm{L}$ & $\mathrm{L}$ & - & - \\
\hline НАТ-P-21 & 1 & 160 & $\mathrm{X}$ & $\mathrm{X}$ & $\mathrm{L}$ & $\mathrm{L}$ & - & - \\
\hline HAT-P-22 & 1 & 298 & $\mathrm{X}$ & $\mathrm{X}$ & $\mathrm{L}$ & $\mathrm{L}$ & - & - \\
\hline HAT-P-23 & 1 & 156 & $\mathrm{X}$ & $\mathrm{X}$ & $\mathrm{L}$ & $\mathrm{L}$ & - & - \\
\hline HAT-P-24 & 2 & 187 & $\mathrm{X}$ & $\mathrm{X}$ & $\mathrm{L}$ & $\mathrm{L}$ & - & - \\
\hline HAT-P-25 & 2 & 124 & $\mathrm{X}$ & $\mathrm{X}$ & $\mathrm{L}$ & $\mathrm{L}$ & - & - \\
\hline HAT-P-26 & 1 & 165 & $\mathrm{X}$ & $\mathrm{X}$ & $\mathrm{L}$ & - & - & - \\
\hline HD 17156 & 1 & 288 & $\mathrm{X}$ & $\mathrm{X}$ & $\mathrm{X}$ & $\mathrm{X}$ & $\mathrm{X}$ & $\mathrm{X}$ \\
\hline HD 80606 & 1 & 357 & $\mathrm{X}$ & $\mathrm{X}$ & $\mathrm{X}$ & $\mathrm{X}$ & $\mathrm{X}$ & $\mathrm{X}$ \\
\hline HD 147506 & 1 & 330 & $\mathrm{X}$ & $\mathrm{X}$ & $\mathrm{X}$ & $\mathrm{X}$ & $\mathrm{X}$ & $\mathrm{X}$ \\
\hline HD 149026 & 4 & 360 & $\mathrm{X}$ & $\mathrm{X}$ & $\mathrm{X}$ & $\mathrm{X}$ & $\mathrm{X}$ & $\mathrm{X}$ \\
\hline HD 189733 & 3 & 337 & $\mathrm{X}$ & $\mathrm{X}$ & $\mathrm{X}$ & $\mathrm{X}$ & $\mathrm{X}$ & $\mathrm{X}$ \\
\hline Kepler-6 & 1 & 97 & $\mathrm{X}$ & $\mathrm{X}$ & $\mathrm{X}$ & $\mathrm{X}$ & $\mathrm{X}$ & $\mathrm{X}$ \\
\hline Kepler-7 & 2 & 69 & $\mathrm{X}$ & $\mathrm{X}$ & $\mathrm{X}$ & $\mathrm{X}$ & $\mathrm{X}$ & $\mathrm{X}$ \\
\hline Kepler-8 & 1 & 85 & $\mathrm{X}$ & $\mathrm{X}$ & $\mathrm{X}$ & $\mathrm{X}$ & $\mathrm{X}$ & $\mathrm{X}$ \\
\hline Kepler-9 & 1 & 63 & $\mathrm{X}$ & - & $\mathrm{X}$ & - & $\mathrm{X}$ & - \\
\hline Kepler-10 & 1 & 226 & $\mathrm{X}$ & $\mathrm{X}$ & $\mathrm{X}$ & $\mathrm{X}$ & $\mathrm{X}$ & $\mathrm{X}$ \\
\hline Kepler-11 & 1 & 41 & $\mathrm{X}$ & - & $\mathrm{X}$ & - & $\mathrm{X}$ & - \\
\hline TRES-1 & 2 & 109 & $\mathrm{X}$ & $\mathrm{X}$ & $\mathrm{X}$ & $\mathrm{X}$ & $\mathrm{L}$ & $\mathrm{X}$ \\
\hline TRES-2 & 2 & 178 & $\mathrm{X}$ & $\mathrm{X}$ & $\mathrm{X}$ & $\mathrm{X}$ & $\mathrm{L}$ & $\mathrm{X}$ \\
\hline TRES-3 & 1 & 97 & $\mathrm{X}$ & $\mathrm{X}$ & $\mathrm{X}$ & $\mathrm{X}$ & $\mathrm{L}$ & $\mathrm{X}$ \\
\hline WASP-1 & 3 & 184 & $\mathrm{X}$ & $\mathrm{X}$ & $\mathrm{X}$ & $\mathrm{X}$ & $\mathrm{X}$ & $\mathrm{X}$ \\
\hline WASP-2 & 1 & 161 & $\mathrm{X}$ & $\mathrm{X}$ & $\mathrm{X}$ & $\mathrm{X}$ & $\mathrm{X}$ & $\mathrm{X}$ \\
\hline WASP-3 & 1 & 252 & $\mathrm{X}$ & $\mathrm{X}$ & $\mathrm{X}$ & $\mathrm{X}$ & $\mathrm{X}$ & $\mathrm{X}$ \\
\hline WASP-12 & 1 & 136 & $\mathrm{X}$ & $\mathrm{X}$ & $\mathrm{X}$ & $\mathrm{X}$ & $\mathrm{X}$ & $\mathrm{X}$ \\
\hline WASP-13 & 1 & 150 & $\mathrm{X}$ & $\mathrm{X}$ & $\mathrm{X}$ & $\mathrm{X}$ & $\mathrm{X}$ & $\mathrm{X}$ \\
\hline WASP-14 & 1 & 265 & $\mathrm{X}$ & $\mathrm{X}$ & $\mathrm{X}$ & $\mathrm{X}$ & $\mathrm{X}$ & $\mathrm{X}$ \\
\hline WASP-17 & 1 & 103 & $\mathrm{X}$ & $\mathrm{X}$ & $\mathrm{X}$ & $\mathrm{X}$ & $\mathrm{X}$ & $\mathrm{X}$ \\
\hline WASP-18 & 1 & 153 & $\mathrm{X}$ & $\mathrm{X}$ & $\mathrm{X}$ & $\mathrm{X}$ & $\mathrm{X}$ & $\mathrm{X}$ \\
\hline WASP-19 & 1 & 100 & $\mathrm{X}$ & $\mathrm{X}$ & $\mathrm{X}$ & $\mathrm{X}$ & $\mathrm{X}$ & $\mathrm{X}$ \\
\hline XO-1 & 1 & 147 & $\mathrm{X}$ & $\mathrm{X}$ & $\mathrm{X}$ & $\mathrm{X}$ & $\mathrm{X}$ & $\mathrm{X}$ \\
\hline $\mathrm{XO}-2$ & 1 & 180 & $\mathrm{X}$ & $\mathrm{X}$ & $\mathrm{X}$ & $\mathrm{X}$ & $\mathrm{X}$ & $\mathrm{X}$ \\
\hline XO-3 & 1 & 243 & $\mathrm{X}$ & $\mathrm{X}$ & $\mathrm{X}$ & $\mathrm{X}$ & $\mathrm{X}$ & $\mathrm{X}$ \\
\hline $\mathrm{XO}-4$ & 1 & 222 & $\mathrm{X}$ & $\mathrm{X}$ & $\mathrm{X}$ & $X$ & $X$ & $\mathrm{X}$ \\
\hline
\end{tabular}

Note. - $\mathrm{U}=$ unconstrained analysis ( $\log g$ free); $\mathrm{C}=$ constrained analysis ( $\log g$ fixed to best value from photometry); $\mathrm{X}$ indicates the method has been applied to this star. $\mathrm{L}=$ results taken from the literature and obtained with the same technique.

spectroscopic analysis.

Sample spectra from each of the instruments are shown in Figure 1.

\section{SPECTROSCOPIC ANALYSIS TECHNIQUES}

Three different techniques were used to determine the spectroscopic parameters $T_{\text {eff }}, \log g$, and [Fe/H]. One procedure was applied to all of our spectra, to provide a homogeneous dataset. The other two techniques were applied to subsets of the spectra in order to evaluate systematic differences in the effective temperatures, surface gravities, and iron abundances. We describe them
TABLE 3

Exoplanet HOST STARS WITH ARCHIVAL FIES SPECTRA.

\begin{tabular}{|c|c|c|c|c|c|c|c|c|}
\hline \multirow[b]{2}{*}{ Star } & \multirow[b]{2}{*}{$N_{\text {obs }}$} & \multirow[b]{2}{*}{ SNR } & \multicolumn{2}{|c|}{$\begin{array}{c}\mathrm{SPC} \\
\text { analysis }\end{array}$} & \multicolumn{2}{|c|}{$\begin{array}{c}\text { SME } \\
\text { analysis }\end{array}$} & \multicolumn{2}{|c|}{$\begin{array}{l}\text { MOOG } \\
\text { analysis }\end{array}$} \\
\hline & & & $\mathrm{U}$ & $\mathrm{C}$ & $\mathrm{U}$ & $\mathrm{C}$ & $\mathrm{U}$ & $\mathrm{C}$ \\
\hline HAT-P-6 & 34 & 87 & $\mathrm{X}$ & $\mathrm{X}$ & - & - & - & - \\
\hline HAT-P-8 & 2 & 121 & $\mathrm{X}$ & $\mathrm{X}$ & - & - & - & - \\
\hline HAT-P-9 & 8 & 32 & $\mathrm{X}$ & $\mathrm{X}$ & - & - & - & - \\
\hline HAT-P-10 & 1 & 24 & $\mathrm{X}$ & $\mathrm{X}$ & - & - & - & - \\
\hline HAT-P-13 & 35 & 92 & $\mathrm{X}$ & $\mathrm{X}$ & - & - & - & - \\
\hline HAT-P-19 & 2 & 37 & $\mathrm{X}$ & $\mathrm{X}$ & - & - & - & - \\
\hline HAT-P-25 & 1 & 21 & $\mathrm{X}$ & $\mathrm{X}$ & - & - & - & - \\
\hline HAT-P-29 & 2 & 36 & $\mathrm{X}$ & $\mathrm{X}$ & - & - & - & - \\
\hline HD 147506 & 1 & 204 & $\mathrm{X}$ & $\mathrm{X}$ & - & - & - & - \\
\hline WASP-1 & 33 & 77 & $\mathrm{X}$ & $\mathrm{X}$ & - & - & - & - \\
\hline WASP-2 & 7 & 86 & $\mathrm{X}$ & $\mathrm{X}$ & - & - & - & - \\
\hline WASP-3 & 9 & 103 & $\mathrm{X}$ & $\mathrm{X}$ & - & - & - & - \\
\hline WASP-10 & 14 & 53 & $\mathrm{X}$ & $\mathrm{X}$ & - & - & - & - \\
\hline WASP-11 & 3 & 59 & $\mathrm{X}$ & $\mathrm{X}$ & - & - & - & - \\
\hline WASP-12 & 22 & 74 & $\mathrm{X}$ & $\mathrm{X}$ & - & - & - & - \\
\hline WASP-13 & 5 & 119 & $\mathrm{X}$ & $\mathrm{X}$ & - & - & - & - \\
\hline WASP-14 & 10 & 152 & $\mathrm{X}$ & $\mathrm{X}$ & - & - & - & - \\
\hline WASP-24 & 5 & 67 & $\mathrm{X}$ & $\mathrm{X}$ & - & - & - & - \\
\hline WASP-31 & 23 & 35 & $\mathrm{X}$ & $\mathrm{X}$ & - & - & - & - \\
\hline WASP-38 & 27 & 203 & $\mathrm{X}$ & $\mathrm{X}$ & - & - & - & - \\
\hline $\mathrm{XO}-3$ & 40 & 98 & $\mathrm{X}$ & $\mathrm{X}$ & - & - & - & - \\
\hline $\mathrm{XO}-4$ & 28 & 59 & $\mathrm{X}$ & $\mathrm{X}$ & - & - & - & - \\
\hline
\end{tabular}

Note. $-\mathrm{U}=$ unconstrained analysis (log $g$ free); $\mathrm{C}=\mathrm{con}-$ strained analysis ( $\log g$ fixed to best value from photometry); $\mathrm{X}$ indicates the method has been applied to this star.

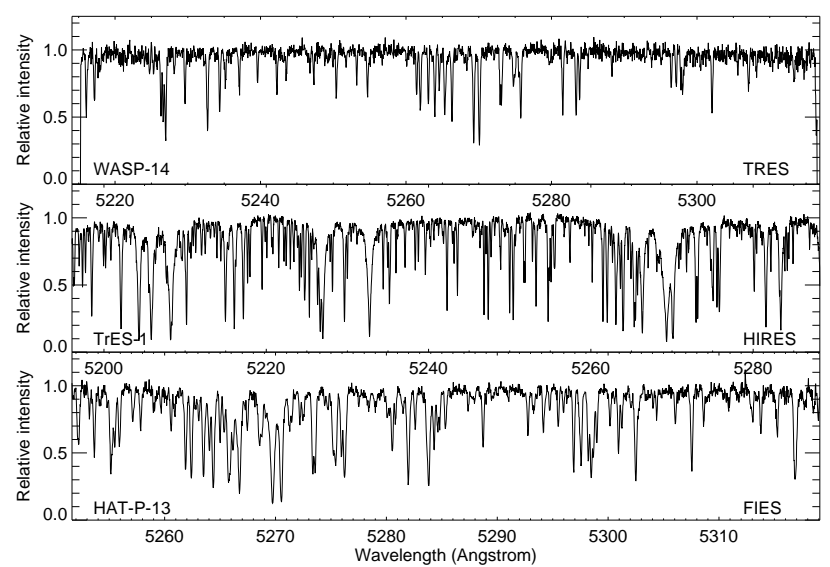

FIG. 1. - Sample spectra near the Mg I b order from each of the three instruments used in this work, as labeled. The signal-to-noise ratios per resolution element are 129 (WASP-14), 109 (TRES-1), and 43 (HAT-P-13).

below. Two of the methods also give the projected rotational velocities, $v \sin i$.

The technique that was applied to all of our TRES, HIRES, and FIES spectra is referred to as Stellar Parameter Classification (SPC), and is described fully by Buchhave et al. (2012). It is based on a crosscorrelation of the observed spectrum against a library of synthetic spectra calculated from Kurucz model atmospheres (Castelli \& Kurucz 2003, 2004), and determines the temperature, surface gravity, metallicity, and projected rotational velocity by seeking the maximum of the cross-correlation coefficient as a function of those parameters (see also Torres et al. 2002; Latham et al. 2002). The grid of synthetic spectra covers a wide range in the above four parameters, although it spans only a limited wavelength region between $5050 \AA$ and $5360 \AA$ 
(Buchhave et al. 2012). This allowed the use of five echelle orders in the FIES spectra, three in the TRES spectra, and two for HIRES. For simplicity, all synthetic spectra in this library were computed with a microturbulent velocity of $\xi=2 \mathrm{~km} \mathrm{~s}^{-1}$ and a macroturbulent velocity of $\zeta_{\mathrm{RT}}=1 \mathrm{~km} \mathrm{~s}^{-1}$. The metallicities derived with this method are not strictly $[\mathrm{Fe} / \mathrm{H}]$, but represent instead an average abundance $[\mathrm{M} / \mathrm{H}]$ of the elements producing absorption features in the spectral region under consideration. Although iron lines tend to dominate, the two indices could be different for a star with peculiar abundances, or more generally for metal-poor stars in which the $\alpha$ elements are often enhanced. However, for the present sample there is no evidence of such anomalies from detailed abundance studies by ourselves or others (e.g., McCullough et al. 2006; Sozzetti et al. 2006; Deleuil et al. 2008; Torres et al. 2008; Anderson et al. 2010a), nor are any of the stars particularly metal-poor so that we would expect $[\alpha / \mathrm{Fe}]$ to be significantly different from zero. In the following we have therefore assumed that $[\mathrm{M} / \mathrm{H}]$ is equivalent to $[\mathrm{Fe} / \mathrm{H}]$.

A second technique was applied to nearly all of the HIRES spectra, and relies on the widely used analysis package Spectroscopy Made Easy (SME; see Valenti \& Piskunov 1996) with the atomic line database of Valenti \& Fischer (2005). The procedure assumes local thermodynamical equilibrium (LTE) and plane parallel geometry, and synthesizes spectra adjusting $T_{\text {eff }}, \log g$, and $[\mathrm{M} / \mathrm{H}]$ to achieve the best match to the observed spectra by minimizing a $\chi^{2}$ function. $[\mathrm{M} / \mathrm{H}]$ is a global abundance parameter in SME that is used to interpolate in the grid of model atmospheres and to scale the solar abundance pattern (except for helium and a few other elements including iron) when calculating opacities. For the present work we report instead the iron abundance, $[\mathrm{Fe} / \mathrm{H}]$, which is a separate variable in the fits. We used eight wavelength segments of approximately $20 \AA$ each, one including the gravity-sensitive $\mathrm{Mg} \mathrm{b}$ triplet and the others spanning the range 6000 $6180 \AA$ A. Following Valenti \& Fischer (2005) we used a fixed value of $\xi=0.85 \mathrm{~km} \mathrm{~s}^{-1}$ for the microturbulent velocity in SME, and the radial-tangential macroturbulent velocity was computed with the prescription given by the same authors. ${ }^{6}$ The performance of this method for deriving effective temperatures, surface gravities, metallicities, and rotational velocities is documented in detail in the same work.

Finally, for about half of the HIRES spectra and some of the TRES spectra we made use of a third method that follows a more classical curve-of-growth approach, as implemented in the spectral synthesis code $\mathrm{MOOG}^{7}$ (Sneden 1973). We used MOOG in conjunction with a grid of Kurucz ATLAS model atmospheres (Kurucz 1993). This technique determines spectroscopic properties under the assumption of LTE, imposing the conditions of excitation and ionization equilibrium. It relies on equivalent widths of selected Fe I and Fe II lines, which we measured either manually or using the automated ARES $^{8}$ tool (Souza et al. 2007). Between 100 and 200

\footnotetext{
6 A sign in their formula for macroturbulence was misprinted the correct expression is $\zeta_{\mathrm{RT}}=3.98+\left(T_{\mathrm{eff}}-5770\right) / 650 \mathrm{~km} \mathrm{~s}^{-1}$.

7 http://www.as.utexas.edu/ chris/moog.html

8 http://www.astro.up.pt/ sousasag/ares/
}

relatively isolated lines were measured in each spectrum, depending on the SNR, with equivalent widths in the range from 2 to $120 \mathrm{~m} \AA$. Microturbulence was determined by imposing the constraint that the Fe I abundance should not depend on the reduced equivalent width. For details of the procedure see, e.g., Sozzetti et al. (2006, 2007). For the TRES spectra the wavelength region used is $\sim 4300-6750 \AA$ (echelle orders 10 to 38 ), and for HIRES we used all orders between $4980 \AA$ and $6420 \AA$.

\section{RESULTS}

\subsection{Unconstrained determinations}

In this section we report spectroscopic determinations of the temperatures, surface gravities, metallicities and projected rotational velocities with SPC, SME, or MOOG in which we solved for those three parameters simultaneously, without making use of any external information about the stars. We refer to these as "unconstrained" results, to distinguish them from the results discussed in the next section in which we hold the surface gravities fixed. The unconstrained results are the most commonly reported in the exoplanet literature, although as we discuss later, the constrained values are generally preferable. For this reason we defer a tabulation of the final parameters until Sect. 4.3. Tables $1,2$. and 3 provide a listing of the method(s) applied to each spectrum. For stars with two or more spectra available from a given telescope we have taken an average of the individual determinations.

A significant fraction of our stars have unconstrained determinations from two or more analysis methods. This allows us to compare results and check for systematic differences, which has seldom been done for transiting planet hosts. In Figure 2 we display the unconstrained values of $T_{\text {eff }}, \log g$, and $[\mathrm{Fe} / \mathrm{H}]$ from the different procedures against each other. For the purpose of this comparison the new SME results obtained here have been augmented with other SME-based results from the literature, relying mostly on HIRES spectra obtained by the HATNet project (Bakos et al. 2004). Those spectra have also been reanalyzed with SPC. The average offsets from at least 30 stars in common between the methods are listed in Table 4, along with the uncertainty of the mean. The average differences are well below $100 \mathrm{~K}$ in temperature, and under 0.1 dex in both $\log g$ and $[\mathrm{Fe} / \mathrm{H}]$. In computing these differences we have adjusted the MOOG abundances to account for a small difference in the adopted solar iron abundance in our implementation of that technique $(A(\mathrm{Fe})=7.52)^{9}$ compared to SPC and $\mathrm{SME}(A(\mathrm{Fe})=7.50)$. On average MOOG is seen to give slightly hotter temperatures and higher surface gravities than both SPC and SME, but lower iron abundances. The SPC results are intermediate between the other two.

A comparison of the projected rotational velocities from SPC and SME is shown in Figure 3. For $v \sin i$ values larger than about $10 \mathrm{~km} \mathrm{~s}^{-1}$ the $\mathrm{SPC}$ values tend to be systematically larger than those from SME. Part of this may be related to differences in the continuumfitting algorithms used in the two techniques (see, e.g., Behr 2003; Royer et al. 2002a, b; Glazunova et al. 2008;

${ }^{9} A(\mathrm{Fe})=\log [N(\mathrm{Fe}) / N(\mathrm{H})]+12$. 

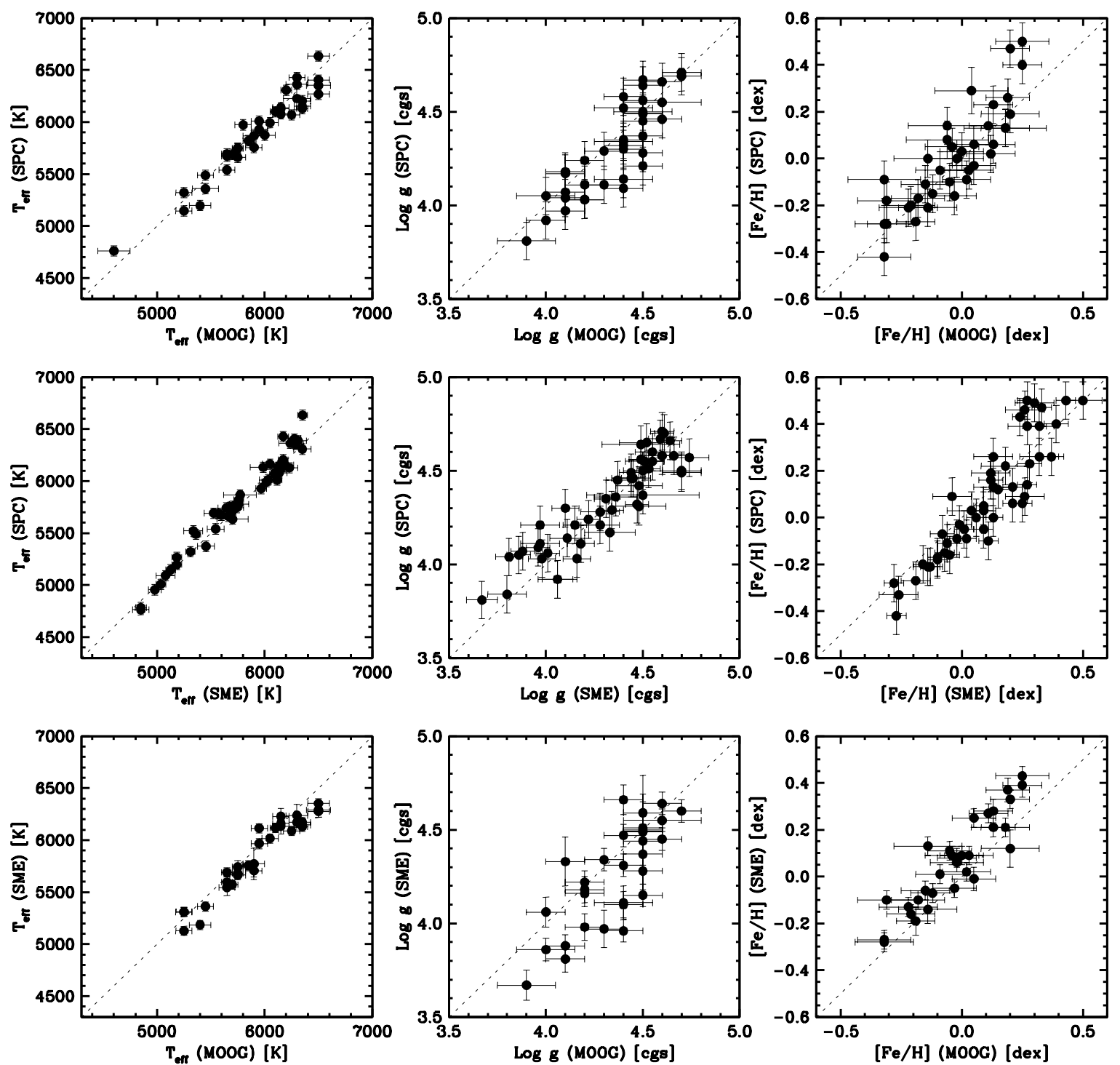

FIG. 2.- Unconstrained spectroscopic results from SPC, SME, and MOOG compared against each other for spectra in common (ranging in number from 31 to 49 , from one or more telescopes; see Table 4). Parity is indicated by the dotted lines.

TABLE 4

COMPARISON OF UNCONSTRAINED RESULTS FOR $T_{\text {eff }}, \log g$, AND

$[\mathrm{FE} / \mathrm{H}]$ FROM DIFFERENT ANALYSIS TECHNIQUES.

\begin{tabular}{lcccc}
\hline \hline & $\Delta T_{\text {eff }}$ & $\Delta \log g$ & $\Delta[\mathrm{Fe} / \mathrm{H}]$ & \\
\multicolumn{1}{c}{ Methods } & $(\mathrm{K})$ & $(\mathrm{dex})$ & $(\mathrm{dex})$ & $N$ \\
\hline SPC-SME & $+32 \pm 12$ & $+0.014 \pm 0.017$ & $-0.023 \pm 0.015$ & 49 \\
SPC-MOOG & $-37 \pm 18$ & $-0.049 \pm 0.020$ & $+0.015 \pm 0.018^{\mathrm{a}}$ & 37 \\
SME-MOOG & $-78 \pm 18$ & $-0.093 \pm 0.030$ & $+0.068 \pm 0.014^{\mathrm{a}}$ & 31 \\
\hline
\end{tabular}

a MOOG metallicities have been adjusted to the same solar iron abundance of $A(\mathrm{Fe})=7.50$ adopted in SPC and SME.

Hensberge et al. 2000).

\subsection{Constrained determinations}

One of the most important uses of the spectroscopic parameters for transiting planet hosts is to infer the mass and radius of the $\operatorname{star}\left(M_{\star}, R_{\star}\right)$, which are needed in turn to establish the mass and radius of the planet. The stellar dimensions are typically obtained by comparing $T_{\text {eff }}, \log g$, and $[\mathrm{Fe} / \mathrm{H}]$ with stellar evolution models, or by using empirical calibrations (Torres et al. 2010a; Enoch et al. 2010). The spectroscopic parameter that most directly affects the determination of the stellar radius is $\log g$, which is a proxy for the luminosity (typically unknown for these stars since the parallaxes have generally not been measured). However, surface gravity has a rather subtle effect on the spectral line profiles, and is difficult to measure accurately. It has been advocated (see Sozzetti et al. 2007; Holman et al. 2007; Torres et al. 


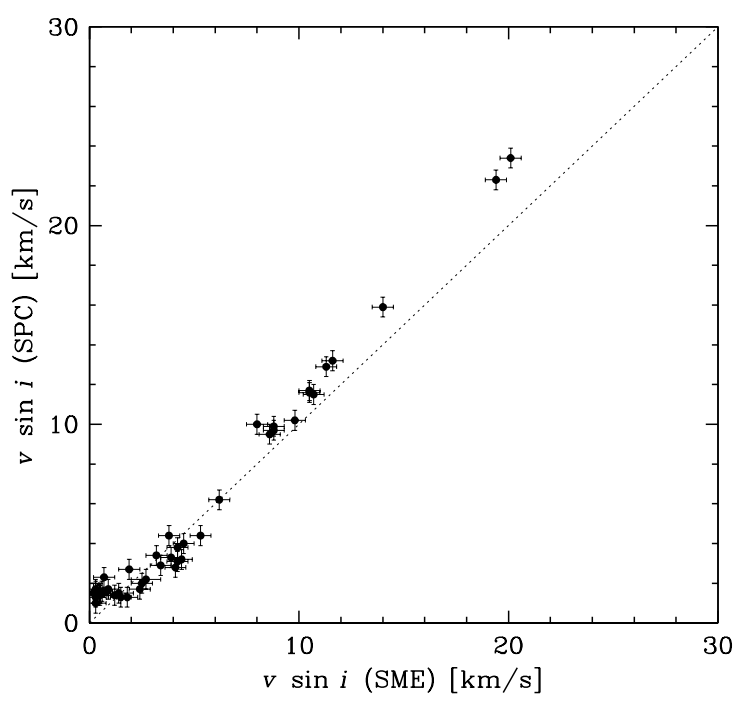

Fig. 3.- Comparison of $v \sin i$ measurements from SPC and SME, based on HIRES spectra.

2008, and others) that a much better constraint on the luminosity of transiting planet hosts can be obtained from the normalized semimajor axis $a / R_{\star}$ that is directly measurable from the transit light curve when the eccentricity is known (Seager \& Mallén-Ornelas 2003). The quantity $a / R_{\star}$ is closely related to the mean stellar density, $\rho_{\star}$. The approach that is now most common in the field for inferring $M_{\star}$ and $R_{\star}$ is to compare $T_{\text {eff }},[\mathrm{Fe} / \mathrm{H}]$, and $\rho_{\star}$ with stellar evolution models, or to use those three quantities as inputs to empirical calibrations. Once the mass and radius are known, a more accurate value of $\log g$ follows trivially. We refer to this as a density-based surface gravity, which is of course model- or calibrationdependent.

The $\log g$ values that emerge from this process do not always agree with the spectroscopic estimates. This inconsistency has usually been attributed to shortcomings in the spectral synthesis techniques. Because the surface gravity is typically correlated with metallicity and temperature in some of the most commonly used methods of analysis, these differences have motivated some authors to repeat the spectroscopic determination of $T_{\text {eff }}$ and $[\mathrm{Fe} / \mathrm{H}]$ holding $\log g$ fixed at the external densitybased values, in order to avoid systematic errors that could propagate into the stellar mass and radius (see, e.g., Kovács et al. 2007; McCullough et al. 2008). We have taken the same approach here, using the best available estimates of the density-based $\log g$ for each system from published photometric analyses. New values of $T_{\text {eff }},[\mathrm{Fe} / \mathrm{H}]$ and $v \sin i$ have been derived with each of the three methodologies, and are presented in Table 5$]^{10}$ The external values of $\log g$ are given later in Sect. 4.3. with our final results.

In Figure 4 we compare the constrained results against the unconstrained values from the previous section, separately for the three methods. For SPC and SME the temperature and the metallicity changes were found to

\footnotetext{
10 For Kepler-9 and Kepler-11 there are no published determinations of $a / R_{\star}$ or $\rho_{\star}$ from which to infer a value of $\log g$ that we can use as a constraint on the spectroscopic determinations. We therefore exclude these stars from Table 5 and subsequent discussion.
}

be strongly correlated with changes in surface gravity, in the sense that $T_{\text {eff }}$ and $[\mathrm{Fe} / \mathrm{H}]$ both increase when a higher gravity is adopted. The slopes of these correlations (determined with simple linear regressions of $Y$ on $X$ using the SLOPES code of Feigelson \& Babu 1992) are such that for an increase of 0.5 dex in $\log g$ the temperatures change systematically by about $+310 \mathrm{~K}$ for SPC and $+350 \mathrm{~K}$ for SME. For a similar increase in $\log g$ the metallicities from SPC and SME both change by about +0.19 dex. These correlations are hardly significant for MOOG: the formal changes per 0.5 dex increase in surface gravity are only $+70 \mathrm{~K}$ and +0.04 dex in $T_{\text {eff }}$ and $[\mathrm{Fe} / \mathrm{H}]$, respectively, which are of the order of the typical internal errors or smaller. We note also that there is some evidence that these correlations depend on temperature, in the sense that for stars cooler than the Sun they are roughly half as large.

SPC and SME are somewhat similar techniques in the sense that they both optimize $T_{\text {eff }}, \log g$, and $[\mathrm{Fe} / \mathrm{H}]$ by seeking the best match between the observed spectrum and a synthetic spectrum. The correlations described above are not completely unexpected in these procedures, as one spectroscopic quantity can play against another to some extent and lead to nearly the same cross-correlation value or $\chi^{2}$ value. For example, stronger lines produced by adopting a higher metallicity for the synthetic spectrum can be compensated for, to first order, by a suitable increase in the effective temperature. A similar degeneracy exists between surface gravity and temperature. In the case of MOOG the effect of these correlations is evidently much weaker. A significant difference between MOOG and the other methods is the line lists. In particular, for this work MOOG uses only Fe I and Fe II lines, whereas SME additionally includes the region of the $\mathrm{Mg} \mathrm{I}$ b triplet, and SPC relies heavily on this same spectral region as well. Therefore for the two latter methods the Mg I b lines contain by far the strongest information on $\log g$. We speculate that any errors in the synthesis of the broad wings of these pressure-sensitive lines may cause errors in the SPC and SME determinations that would likely also impact the $T_{\text {eff }}$ and $[\mathrm{Fe} / \mathrm{H}]$ values because of the correlations described above; MOOG, on the other hand, would be unaffected. While we have no evidence of such errors at the present time, this could be investigated by using different atmospheric models. We note also that the surface gravity in the unconstrained MOOG analysis is determined implicitly by requiring that the iron abundance for Fe I and Fe II from the measured equivalent widths be the same (ionization equilibrium). When fixing $\log g$ to a value determined externally this condition is generally no longer met, although the discrepancy for our sample (about 0.05 dex, on average) is small compared to the nominal uncertainty in the Fe I abundance. This lack of ionization equilibrium seems to have little effect on the other parameters, and could be a sign of real differences between Fe I and Fe II (see, e.g., Schuler et al. 2010, and references therein), or deficiencies in the models (Yong et al. 2004). For all three methods we find that the goodness of fit (as quantified by $\chi^{2}$ or the average cross-correlation coefficient) is only slightly lower in the constrained fits compared to those in which $\log g$ is left free.

Differences between the results with unconstrained surface gravities and those using the density-based values 
TABLE 5

Spectroscopic Results using the external CONStraint on $\log g$ From Photometry (CONSTRAined Analysis).

\begin{tabular}{|c|c|c|c|c|c|c|c|c|c|}
\hline \multirow[b]{2}{*}{ Star } & \multicolumn{3}{|c|}{ SPC Analysis } & \multicolumn{3}{|c|}{ SME Analysis } & \multicolumn{2}{|c|}{ MOOG Analysis } & \multirow[b]{2}{*}{$\mathrm{Tel}^{\mathrm{a}}$} \\
\hline & $\begin{array}{l}T_{\text {eff }} \\
(\mathrm{K})\end{array}$ & $\begin{array}{c}{[\mathrm{Fe} / \mathrm{H}]} \\
(\mathrm{dex})\end{array}$ & $\begin{array}{c}v \sin i \\
\left(\mathrm{~km} \mathrm{~s}^{-1}\right)\end{array}$ & $\begin{array}{l}T_{\text {eff }} \\
(\mathrm{K})\end{array}$ & $\begin{array}{c}{[\mathrm{Fe} / \mathrm{H}]} \\
(\mathrm{dex})\end{array}$ & $\begin{array}{c}v \sin i \\
\left(\mathrm{~km} \mathrm{~s}^{-1}\right)\end{array}$ & $\begin{array}{l}T_{\text {eff }} \\
(\mathrm{K})\end{array}$ & $\begin{array}{c}{[\mathrm{Fe} / \mathrm{H}]} \\
(\mathrm{dex})\end{array}$ & \\
\hline CoRoT-1 & $6280 \pm 50$ & $-0.04 \pm 0.08$ & $4.3 \pm 0.5$ & $6312 \pm 44$ & $+0.08 \pm 0.04$ & $4.9 \pm 0.5$ & $6300 \pm 75$ & $+0.05 \pm 0.11$ & $\mathrm{H}$ \\
\hline CoRoT-2 & $5552 \pm 50$ & $-0.15 \pm 0.08$ & $10.6 \pm 0.5$ & $5602 \pm 44$ & $-0.01 \pm 0.04$ & $10.0 \pm 0.5$ & $5550 \pm 75$ & $-0.10 \pm 0.11$ & $\mathrm{H}$ \\
\hline CoRoT-7 & $5392 \pm 50$ & $+0.08 \pm 0.08$ & $0.5 \pm 0.5$ & $5274 \pm 44$ & $+0.02 \pm 0.04$ & $0.5 \pm 0.5$ & $5250 \pm 75$ & $0.00 \pm 0.08$ & $\mathrm{H}$ \\
\hline НАТ-P-3 & $5201 \pm 50$ & $+0.45 \pm 0.08$ & $1.8 \pm 0.5$ & $\ldots$ & $\ldots$ & $0.5 \pm 0.5$ & $\ldots$ & $\ldots$ & $\mathrm{H}$ \\
\hline НАТ-P-3 & $5247 \pm 50$ & $+0.36 \pm 0.08$ & $2.2 \pm 0.5$ & $\ldots$ & $\ldots$ & $\ldots$ & $\ldots$ & $\ldots$ & $\mathrm{T}$ \\
\hline
\end{tabular}

Note. - Table 5 is published in its entirety in the electronic edition of ApJ. A portion is shown here for guidance regarding its form and content. Errors are internal.

Telescope/instrument combination: $\mathrm{H}=$ Keck/HIRES, $\mathrm{F}=$ NOT/FIES, $\mathrm{T}=\mathrm{FLWO} / \mathrm{TRES}$.
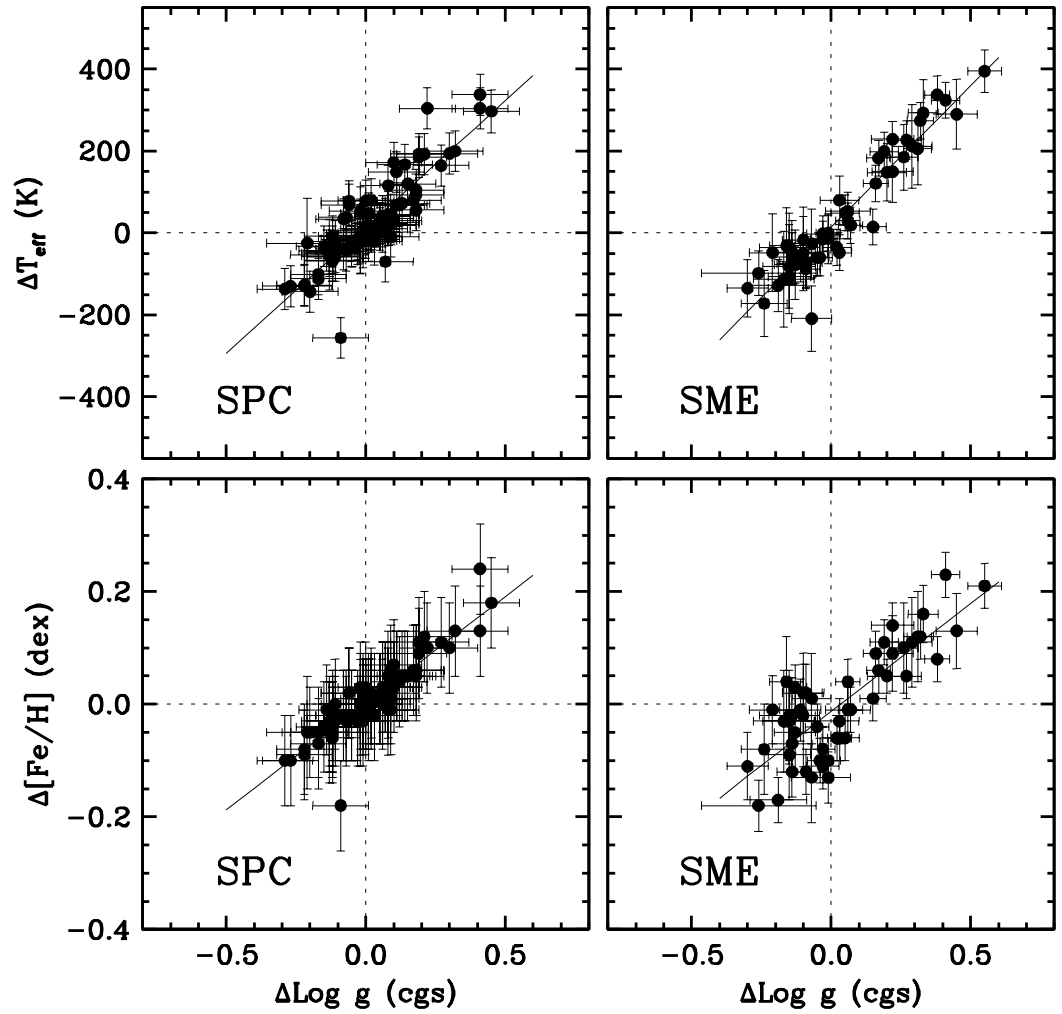

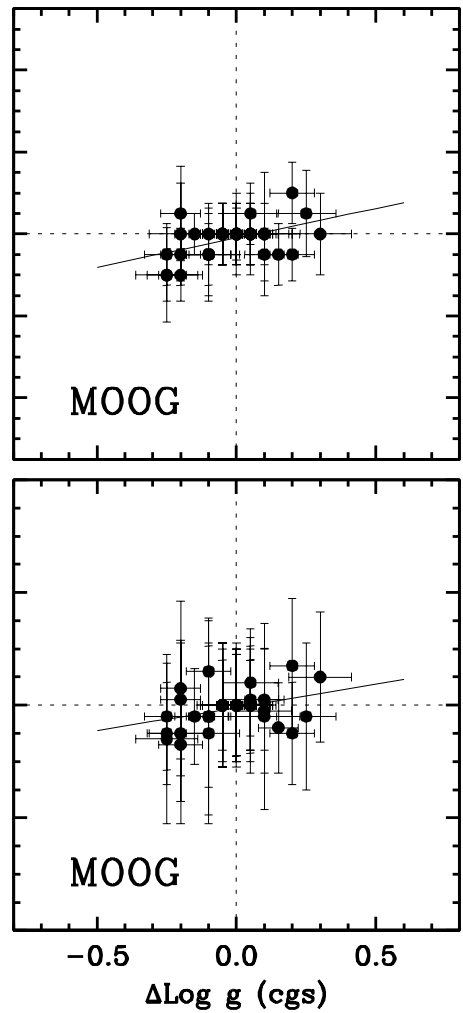

FIG. 4.- Impact on the temperatures and metallicities of fixing $\log g$ to the photometric values, for each of the three methods. The panels show the differences in the sense 〈constrained minus unconstrained $\rangle$ as a function of the change in log $g$. The lines represent linear fits computed with the $\operatorname{OLS}(Y \mid X)$ procedure as implemented in the SLOPES code of Feigelson \& Babu (1992). The Pearson correlation coefficients in $\Delta T_{\text {eff }}$ vs. $\Delta \log g$ and $\Delta[\mathrm{Fe} / \mathrm{H}]$ vs. $\Delta \log g$ range from 0.82 to 0.95 for SPC and SME, but are only 0.49 and 0.37 for MOOG.

were found to be up to 0.5 dex for SPC and SME, and up to 0.3 dex for MOOG. To the extent that the photometric constraints on $\rho_{\star}$ (and therefore $\log g$ ) are accurate, this suggests that all three spectroscopic procedures are vulnerable to systematics, though perhaps not to the same degree or for the same reasons.

Indirect evidence of these effects may perhaps be seen already in published results from the two surveys that have produced the majority of the transiting planet discoveries from the ground: the WASP project (Pollacco et al. 2006) and the HATNet project (Bakos et al. 2004). The HATNet group has generally used SME for the spectroscopic analysis of the parent stars, and in most cases the studies have been iterated as described above, using the constraint from the transit light curves to set $\log g$. The WASP team has occasionally also used SME, although more recently they have relied on the UCLSYN package (Smalley et al. 2001) and in general their spectroscopic results are from an unconstrained analysis ( $\log g$ free).

A comparison of the distribution of published metallicities for the host stars from these two groups is seen in Figure 5 . The average metallicities differ by about 0.17 dex, and a Kolmogorov-Smirnov test suggests the distributions are statistically different, with a false alarm probability (FAP) of $0.14 \%$. Both surveys are magnitude limited, with the WASP program being typically shallower, so a difference in the metallicity distributions is possible in principle. However, we find that the mean visual magnitude of the two star samples is essentially the same ( $V \approx 11.5$ for WASP and $V \approx 11.3$ for HATNet), and a K-S test indicates the brightness distributions are indistinguishable $(\mathrm{FAP}=0.259)$. It seems likely, therefore, that the difference in the average metallicities is 


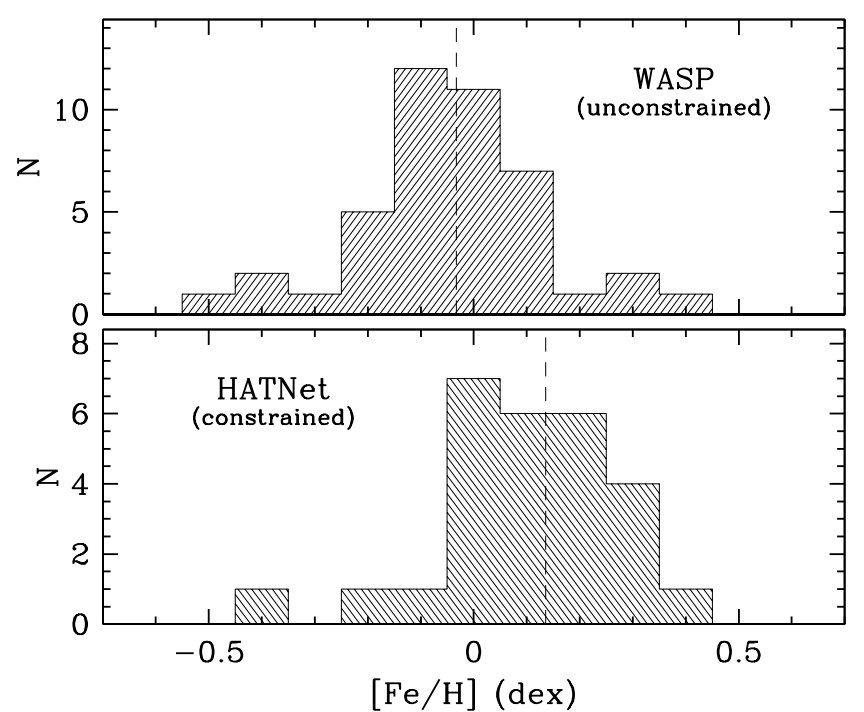

FIG. 5.- Metallicity distributions for transiting planet hosts analyzed by the WASP and HATNet groups (43 and 27 stars, respectively). The results for the WASP stars are based on unconstrained spectroscopic analyses, while those from HATNet rely on $\log g$ as constrained photometrically by the mean stellar density The mean of each distribution is indicated with a dashed line.
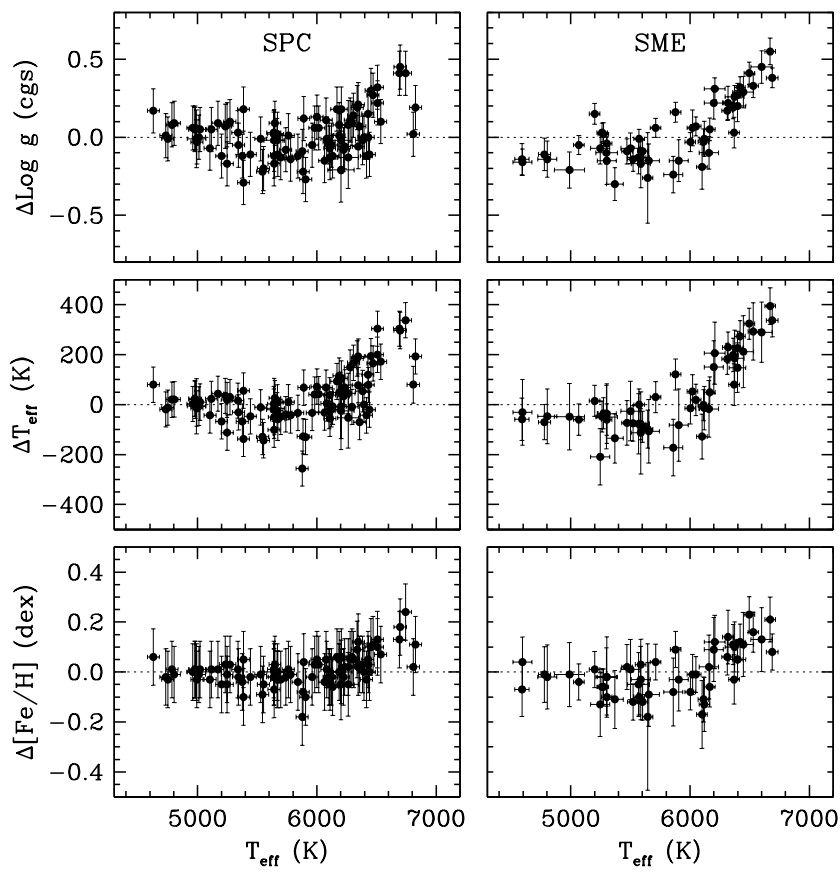

FIG. 6.- Constrained minus unconstrained differences in surface gravity, effective temperature, and iron abundance $\left(\Delta \log g, \Delta T_{\text {eff }}\right.$, and $\Delta[\mathrm{Fe} / \mathrm{H}]$ ) as a function of effective temperature, for SPC (left panels) and SME (right).

mostly due to the analysis. It goes in the direction expected, as the constrained $\log g$ values adopted by the HATNet team are more often larger than the unconstrained values, which according to Figure 4 should lead to higher metallicities in that survey, just as observed.

Figure 6 shows that for SPC and SME the differences between the constrained and unconstrained results for $T_{\text {eff }}, \log g$, and $[\mathrm{Fe} / \mathrm{H}]$ are a function of the temperature of the star. In the case of SME the external surface gravities for stars up to roughly $6000 \mathrm{~K}$ tend to be lower than the unconstrained values, on average, and in turn lead to

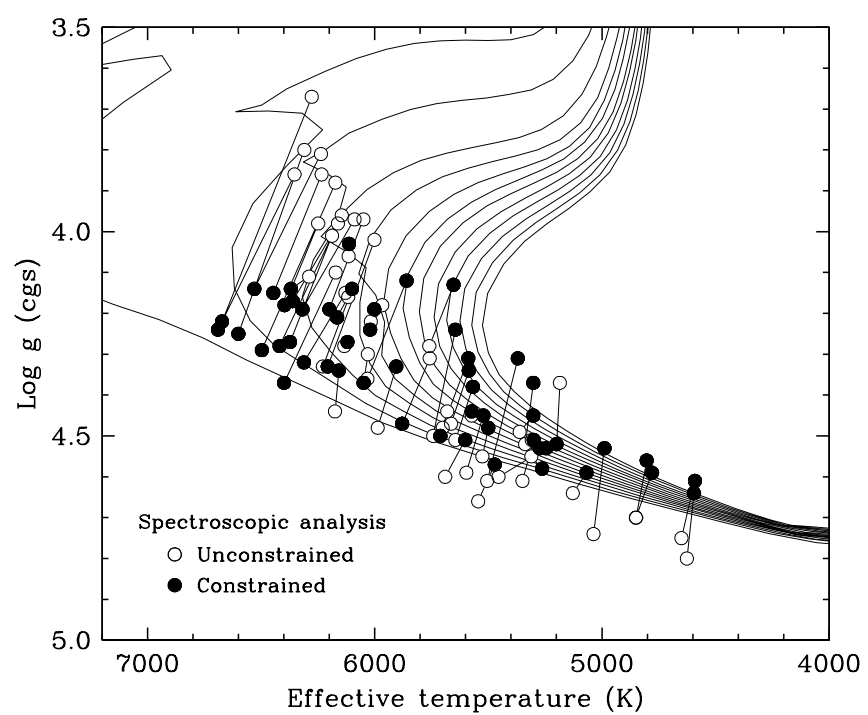

FIG. 7.- Illustration of the impact that constraining $\log g$ has on the location of transiting planet host stars in the H-R diagram, shown here for spectra analyzed with SME. We include additional stars with SME-based results from the literature carried out in the same way as in this work. Unconstrained and constrained results are connected with lines, and shown against representative stellar evolution models from Yi et al. (2001) for solar metallicity, and ages between 1 and 13 Gyr (in steps of 1 Gyr).

systematically lower temperatures and metallicities. For hotter stars the trend reverses sharply, reaching maximum differences of 0.5 dex in $\log g, 400 \mathrm{~K}$ in $T_{\text {eff }}$, and 0.2 dex in metallicity compared to those with $\log g$ free. For SPC the external $\log g$ values differ somewhat less from the unconstrained determinations, but again the hotter stars give higher temperatures and $[\mathrm{Fe} / \mathrm{H}]$ values when imposing this constraint. No such dependence is apparent for the MOOG results. We also found no significant dependence of $\Delta T_{\text {eff }}, \Delta \log g$, or $\Delta[\mathrm{Fe} / \mathrm{H}]$ with either metallicity or surface gravity, in any of the three methods. The projected rotational velocities from SPC and SME are insensitive to the change in $\log g$.

As mentioned above the trends in SPC and SME with effective temperature are most likely related to the fact that the information on surface gravity in these methods comes almost exclusively from the pressure-broadened wings of the $\mathrm{Mg} \mathrm{Ib}$ lines at $\sim 5200 \AA$. For stars hotter than about $6000 \mathrm{~K}$ the wings of these lines weaken considerably, and the methods lose sensitivity to $\log g$. It is less clear why the bias in $\log g$ for such stars is toward smaller values, as opposed to being toward higher values or simply having a larger scatter. In any case, the positive correlation between $\log g, T_{\text {eff }}$, and $[\mathrm{Fe} / \mathrm{H}]$ explains the upturn in the last two quantities in Figure 6 .

As a result of the temperature correlation for SPC and SME shown in Figure 6] the net effect of applying the $\log g$ constraint with those methods is to shift the stars in the H-R diagram towards a less evolved state (closer to the zero-age main sequence). The less evolved state also seems more likely a priori, because of the slower speed of evolution for unevolved stars. We illustrate this for SME in Figure 7 . The trend has a significant impact on the inferred radii of the stars, which we discuss more quantitatively in Sect. 6 .

The constrained results from the different methods are compared in Figure 8 for stars in common. Our expecta- 

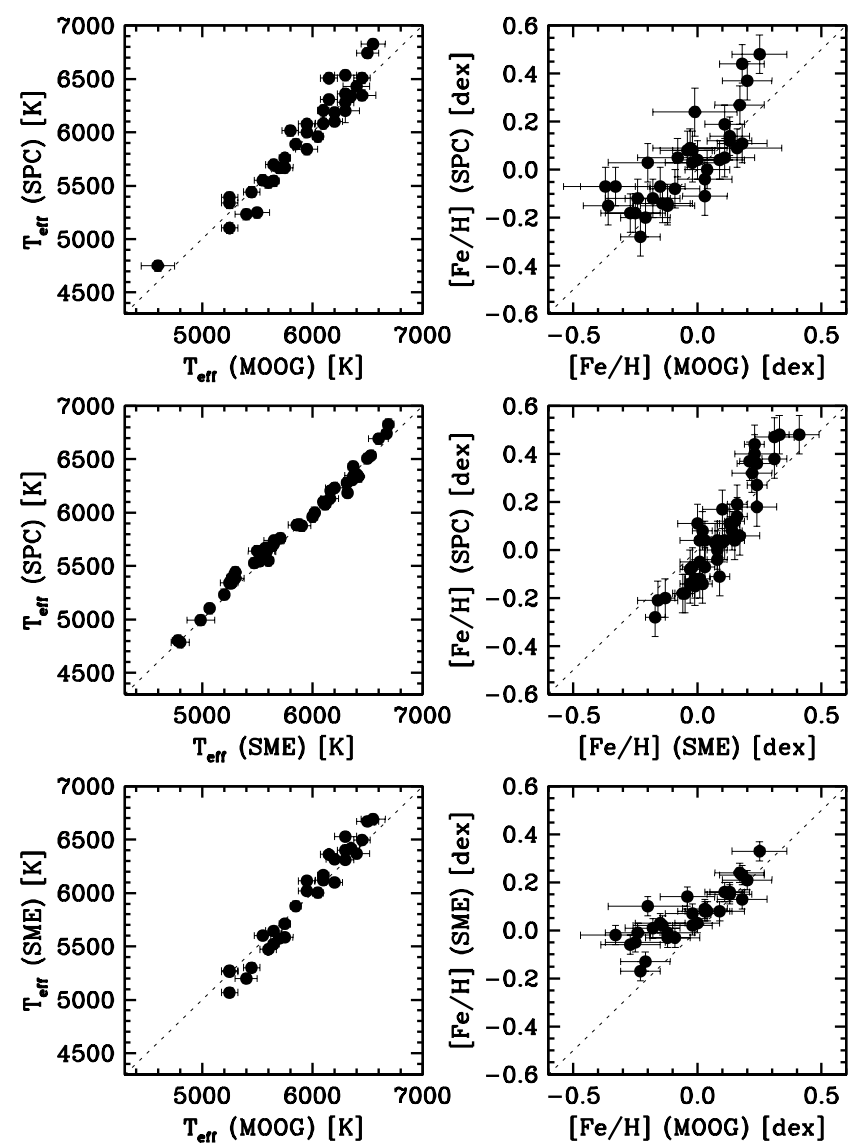

FIG. 8.- Constrained spectroscopic results from SPC, SME, and MOOG compared against each other for spectra in common (ranging from 29 to 44, from one or more telescopes; see Table 6). One-to-one relations are indicated with dotted lines.

tion was that the methods would show better agreement than the unconstrained results, since some of the biases in the determination of $T_{\text {eff }}$ and $[\mathrm{Fe} / \mathrm{H}]$ that are intrinsic to each of the techniques would be reduced by enforcing an external constraint on $\log g$. This is indeed the case, although residual trends can still be seen in some of the panels of Figure 8, such as between MOOG and $\mathrm{SME}$ in $[\mathrm{Fe} / \mathrm{H}]$, or between SPC and SME in temperature and also metallicity. These are most likely a reflection of strong correlations between the spectroscopic parameters that are present in at least two of the methods (SPC and $\mathrm{SME}$ ), as already described, and the different degrees to which the three procedures respond to the imposition of an external $\log g$ constraint. Many details of the spectroscopic analysis are likely to influence the results in ways that are difficult to predict or quantify. This includes the accuracy of the continuum normalization of the spectra, the model atmospheres used, the adopted line lists, and the sensitivity to the SNR of the spectra, which varies from method to method. Nevertheless, average differences between the final values from SPC, SME, and MOOG remain quite small, as seen in Table 6 .

\subsection{Final spectroscopic results}

The sensitivity of SPC and SME to changes in the surface gravity suggests these techniques are more susceptible to systematic bias than MOOG, although $\log g$ errors up to 0.3 dex occur even with MOOG. The smaller impact of those errors on $T_{\text {eff }}$ and $[\mathrm{Fe} / \mathrm{H}]$ in the latter
TABLE 6

COMPARISON OF CONSTRAINED RESUlts FOR $T_{\text {eff }}$ AND $[\mathrm{FE} / \mathrm{H}]$ FROM DIFFERENT ANALYSIS TECHNIQUES.

\begin{tabular}{lccc}
\hline \hline Methods & $\Delta T_{\text {eff }}$ & $\Delta[\mathrm{Fe} / \mathrm{H}]$ \\
$(\mathrm{K})$ & $(\mathrm{dex})$ & $N$ \\
\hline SPC-SME & $+27 \pm 9$ & $-0.020 \pm 0.015$ & 44 \\
SPC-MOOG & $+27 \pm 23$ & $+0.049 \pm 0.019^{\mathrm{a}}$ & 36 \\
SME-MOOG & $+8 \pm 22$ & $+0.081 \pm 0.017^{\mathrm{a}}$ & 29 \\
\hline
\end{tabular}

${ }^{a}$ MOOG metallicities have been adjusted to the same solar iron abundance of $A(\mathrm{Fe})=7.50$ adopted in SPC and SME.

method does not necessarily guarantee those quantities are free from bias. It is difficult to ascertain whether the results from one technique are more accurate than another. Independent checks on the temperatures could in principle be obtained from the measurement of bolometric fluxes and precise interferometric angular diameters, but the stars in the present sample are generally too faint for current long-baseline interferometers. Photometric temperature estimates based on color indices might provide an alternative, but suffer from the possibility of reddening, which is unknown for most of these stars.

Given that the average systematic differences in Table 6 between the three methodologies are relatively small, the final spectroscopic parameters we adopted for stars analyzed with more than one method or observed using more than one instrument is the weighted mean, which should not significantly affect the homogeneity of the results. We collect the final parameters for 56 transiting planet hosts in Table 7, where the uncertainties include a contribution added in quadrature from the overall scatter of the measurements available for stars with multiple determinations. These added dispersions are $\sigma_{T_{\text {eff }}}=59 \mathrm{~K}, \sigma_{[\mathrm{Fe} / \mathrm{H}]}=0.062 \mathrm{dex}$, and $\sigma_{v \sin i}=0.85 \mathrm{~km} \mathrm{~s}^{-1}$. The table also reports the external constraint on $\log g$ used for the second iteration of the spectroscopic analyses with SPC, SME, and MOOG.

\section{COMPARISON WITH OTHER DETERMINATIONS}

Prior to this study, the largest effort to determine spectroscopic parameters for transiting planet hosts in a homogeneous way was that of Ammler-von Eiff et al. (2009), who used MOOG following the precepts of Santos et al. (2004, 2006). Our sample and theirs have 14 stars in common.

A comparison of our results from Table 7 with theirs is seen in Figure 9, where the differences in $T_{\text {eff }},[\mathrm{Fe} / \mathrm{H}]$, and $\log g$ for these 14 stars are plotted in the sense $\langle$ Ammlervon Eiff minus Table 7). As indicated earlier our adopted surface gravities are the density-based values, not the spectroscopic values. The temperatures on the horizontal axis of the figure are from our own determinations.

Although the sample is small, there appears to be a significant systematic difference in the surface gravities that correlates with temperature (bottom panel), such that Ammler-von Eiff et al. (2009) measure a higher $\log g$ for the hotter stars. It is difficult to see how a bias in our $\log g$ values from Table 7 could cause this, as those determinations rest essentially on the $a / R_{\star}$ measurements 
TABLE 7

Final SpeCtroscopic Results (aVERAge of all NeW Determinations).

\begin{tabular}{ccccccc}
\hline \hline Name & $\begin{array}{c}T_{\text {eff }} \\
(\mathrm{K})\end{array}$ & $\begin{array}{c}{[\mathrm{Fe} / \mathrm{H}]} \\
(\mathrm{dex})\end{array}$ & $\begin{array}{c}v \sin i \\
\left(\mathrm{~km} \mathrm{~s}^{-1}\right)\end{array}$ & $N^{\mathrm{a}}$ & $\begin{array}{c}\text { External } \\
\log g(\mathrm{cgs})\end{array}$ & Source $^{\mathrm{b}}$ \\
\hline CoRoT-1 & $6298 \pm 66$ & $+0.06 \pm 0.07$ & $4.6 \pm 0.9$ & 3,2 & $4.33 \pm 0.01$ & 1 \\
CoRoT-2 & $5575 \pm 66$ & $-0.04 \pm 0.08$ & $10.3 \pm 0.9$ & 3,2 & $4.51 \pm 0.04$ & 2 \\
CoRoT-7 & $5313 \pm 73$ & $+0.03 \pm 0.07$ & $0.5 \pm 1.0$ & 3,1 & $4.54 \pm 0.04$ & 3 \\
HAT-P-3 & $5224 \pm 69$ & $+0.41 \pm 0.08$ & $1.5 \pm 1.0$ & 2,3 & $4.58 \pm 0.03$ & 4 \\
HAT-P-4 & $5890 \pm 67$ & $+0.20 \pm 0.08$ & $5.6 \pm 0.9$ & 3,3 & $4.14 \pm 0.03$ & 5 \\
\hline
\end{tabular}

Note. - Table 17 is published in its entirety in the electronic edition of ApJ. A portion is shown here for guidance regarding its form and content.

${ }^{a}$ Number of determinations for $T_{\text {eff }}$ and $[\mathrm{Fe} / \mathrm{H}]$ (based on spectra from different telescopes, or derived with different methodologies), followed by the number of determinations for $v \sin i$.

b Sources for the external $\log g$ constraint (either reported directly in these studies, or inferred from $M_{\star}$ and $R_{\star}$ as reported there, or based on the reported $a / R_{\star}$ values and our own stellar evolution modeling using isochrones from Yi et al. 2001). References: (1) Bean (2009); (2) Gillon et al. (2010); (3) Léger et al. (2009); (4) Torres et al. (2007); (5) Kovács et al. (2007); (6) Bakos et al. (2007); (7) Noves et al. (2008); (8) Christensen-Dalsgaard et al. (2010); (9) Latham et al. (2009); (10) Shporer et al. (2009); (11) West et al. (2009); (12) Bakos et al. (2010); (13) Bakos et al. (2009); (14) Torres et al. (2010b); (15) Kovács et al. (2010); (16) Buchhave et al. (2010b);

(17) Howard et al. (2012); (18) Hartman et al. (2011a); (19) Bakos et al. (2011);

(20) Kipping et al. (2010); (21) Quinn et al. (2012); (22) Hartman et al. (2011b);

(23) Buchhave et al. (2011); (24) Gilliland et al. (2011); (25) Hébrard et al. (2010); (26) Pál et al. (2010); (27) Torres et al. (2008); (28) Kipping \& Bakos (2011a); (29) Batalha et al. (2011); (30) Kipping \& Bakos (2011b); (31) Sozzetti et al. (2009);

(32) Simpson et al. (2010); (33) Christian et al. (2009); (34) Enoch et al. (2010); (35) Skillen et al. (2009); (36) Joshi et al. (2009); (37) Anderson et al. (2010a) (38) Southworth et al. (2009b); (39) Anderson et al. (2010b); (40) Street et al. (2010); (41) Anderson et al. (2011); (42) Barros et al. (2011); (43) Burke et al. (2010); (44) Fernandez et al. (2009); (45) Winn et al. (2008); (46) Narita et al. (2010).
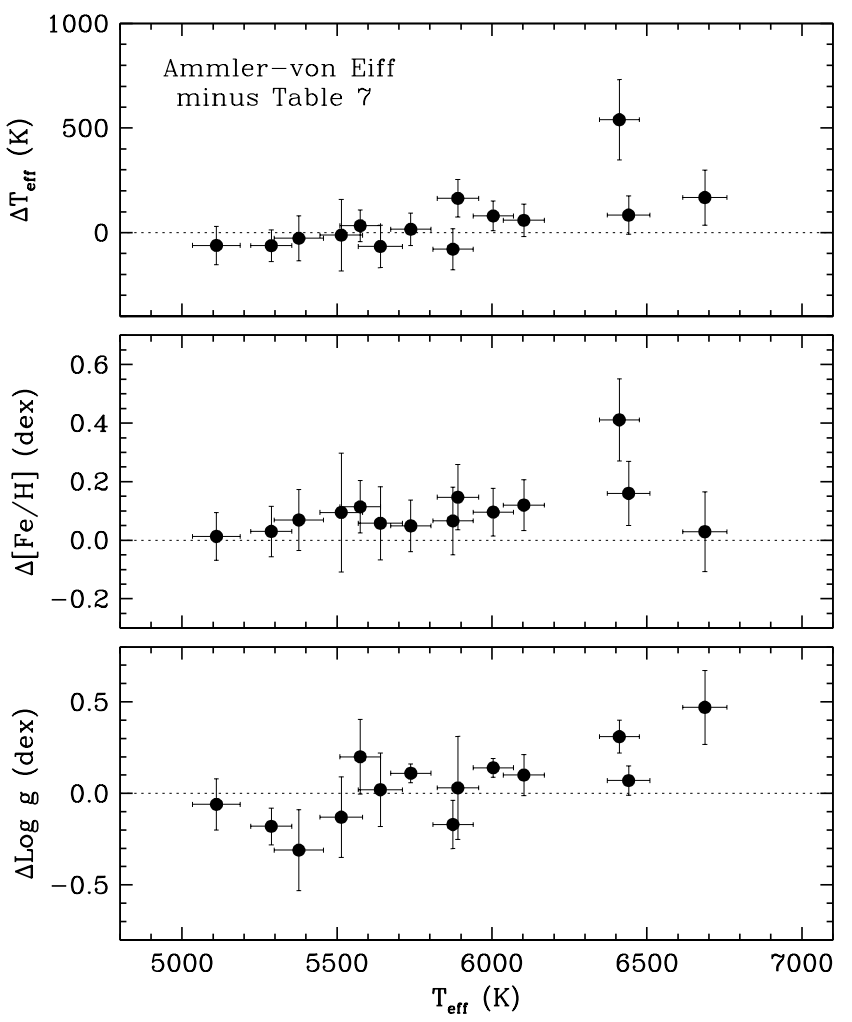

FIG. 9.- Comparison of our results as listed in Table 7 with those of Ammler-von Eiff et al. (2009), for the 14 stars in common. The temperature, metallicity, and gravity differences are in the sense "theirs minus ours", where our $\log g$ values are the density-based determinations. The temperatures plotted on the horizontal scale are our own. The high value in the top two panels corresponds to HD 147506 (HAT-P-2). from the transit light curves. There may also be a trend of $\Delta T_{\text {eff }}$ with temperature (top panel), though it seems marginal. For the metallicities there is no significant correlation with temperature, but we note that all of the Ammler-von Eiff et al. (2009) values of $[\mathrm{Fe} / \mathrm{H}]$ are higher than ours, the average difference being $+0.10 \pm 0.03$ dex.

\section{IMPACT OF THE SURFACE GRAVITY CONSTRAINT ON STELLAR MASSES AND RADII}

In Figure 7 above we presented a graphical illustration of how the inferred evolutionary state of the star can change considerably as a result of applying the external (photometric) constraint on $\log g$ in the spectroscopic analysis. As might be expected, this can also lead to significant changes in the mass and radius derived for the star, which are of more immediate interest for computing planetary properties in transiting planet systems.

To explore this more quantitatively we have derived stellar masses and radii for the same stars shown in Figure 7 following the common procedure of comparing the spectroscopic quantities $T_{\text {eff }}, \log g$, and $[\mathrm{Fe} / \mathrm{H}]$ against stellar evolution models. For each star we performed a Monte Carlo simulation in which we drew 10,000 values of the three spectroscopic parameters from Gaussian distributions characterized by the measured values and observational errors, assuming they are uncorrelated. We compared each set with isochrones from the Yonsei-Yale series by Yi et al. (2001), seeking the best match in a $\chi^{2}$ sense. Ages were allowed to vary in steps of $0.1 \mathrm{Gyr}$ over the range from 0.1 to $13.7 \mathrm{Gyr}$, and the $\alpha$-element abundance was assumed to be solar for this test $([\alpha / \mathrm{Fe}]$ $=0.0)$. Stellar masses and radii were determined from the mode of the respective posterior probability distri- 

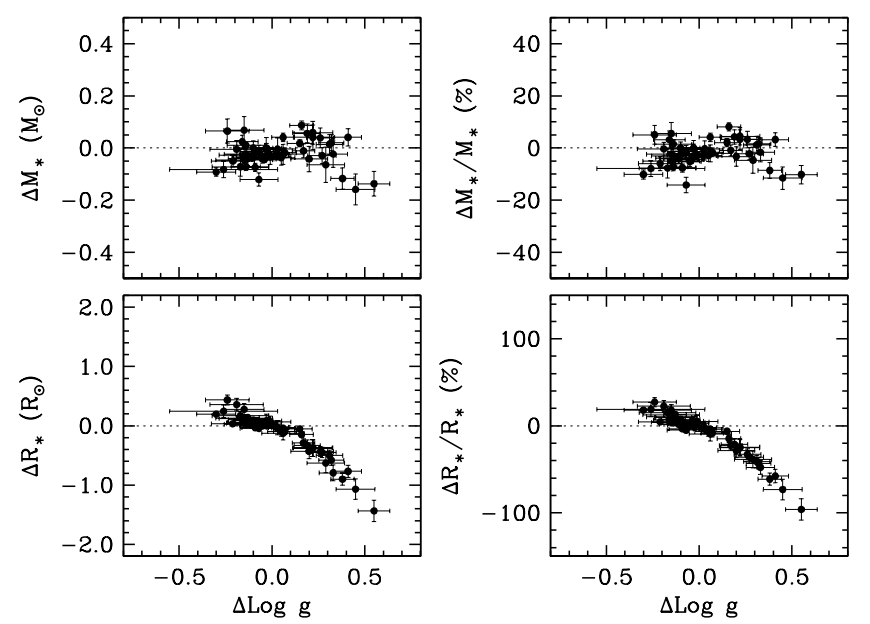

FIG. 10. - Mass and radius differences resulting from the use of constrained and unconstrained spectroscopic properties from SME along with stellar evolution models. Differences in the sense $\langle$ constrained minus unconstrained $\rangle$ are shown in absolute units on the left, and as a percentage of $M_{\star}$ or $R_{\star}$ on the right.

butions, and 1- $\sigma$ lower and upper confidence limits were defined by the $15.85 \%$ and $84.15 \%$ percentiles of the cumulative distribution. We carried out these calculations first using the unconstrained SME results, and then repeated the process with the constrained results.

A comparison between $M_{\star}$ and $R_{\star}$ from these two sets of parameters is shown in Figure 10 as a function of $\Delta \log g$, where the changes are displayed both in absolute units and as a percentage. In the most extreme cases the unconstrained radii can be off by up to $100 \%$. This occurs for the hotter stars, as can be seen in Figure 7 The impact on the mass is also not negligible, reaching $\sim 15 \%$ in some cases. Errors of this magnitude are almost always larger than other observational errors. Very similar results were obtained using the SPC determinations.

The importance of this bias coming from the reliance on the weakly determined spectroscopic $\log g$ is now widely recognized in the community, and is largely avoided in current analyses of transiting planets by adopting instead the density-based $\log g$ to infer $M_{\star}$ and $R_{\star}$. However, many of those same studies still retain the temperatures and metallicities from unconstrained spectroscopic analyses in which the surface gravity was left free. Because $T_{\text {eff }}$ and $[\mathrm{Fe} / \mathrm{H}]$ typically suffer from strong correlations, as shown earlier in Figure 4, those values have a residual bias that is often not negligible and can propagate to the stellar masses and radii. This bias has been generally overlooked.

We have quantified this systematic effect by repeating the determination of $M_{\star}$ and $R_{\star}$ for the same sample above using the photometry-based $\log g$, but deliberately adopting $T_{\text {eff }}$ and $[\mathrm{Fe} / \mathrm{H}]$ from the unconstrained spectroscopic analysis, to emulate the procedure often followed in published studies. Figure 11 compares these masses and radii with those based on our second iteration of SME, in which the temperature and metallicity were redetermined using the external $\log g$ constraint. There are clear differences in the stellar properties that mimic those seen in Figure 6. We conclude that even if a more accurate $\log g$ is adopted for inferring $M_{\star}$ and $R_{\star}$ from evolutionary models, using the unconstrained values of the temperatures and metallicities rather than

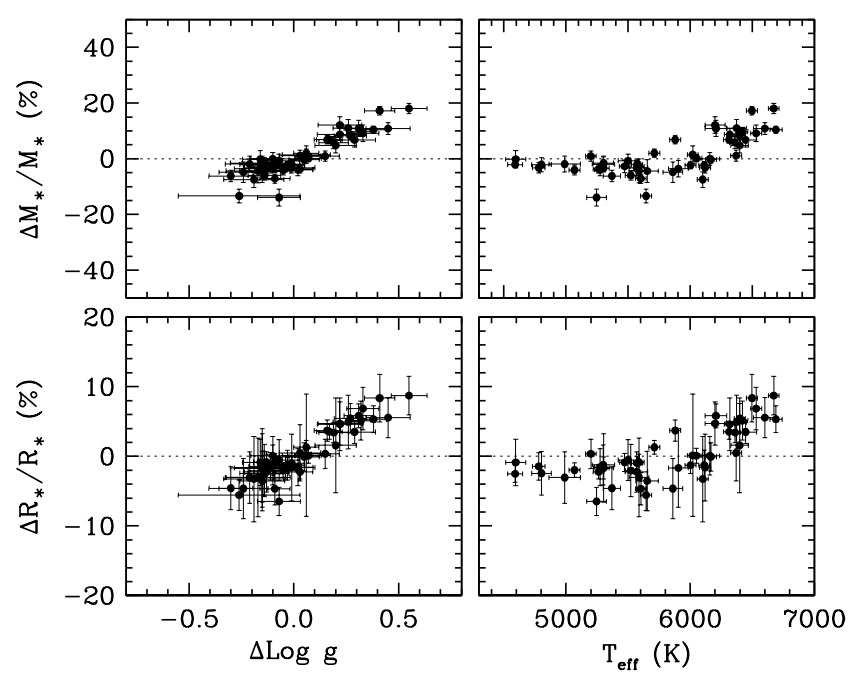

FIG. 11. - Systematic errors in the stellar mass and radius (expressed as a percentage) when using unconstrained values of $T_{\text {eff }}$ and $[\mathrm{Fe} / \mathrm{H}]$ from SME together with the external photometric constraint on $\log g$ from the mean stellar density. The differences shown are between the mixed usage just mentioned and the constrained results from a second iteration of SME described in the text, in the sense $\langle$ mixed minus constrained $\rangle$.

those from a second iteration of the spectroscopic analysis can still lead to stellar masses that are up to $20 \%$ too large, and radii that are overestimated by as much as $10 \%$ for the hotter stars. Since planetary properties from Doppler and light-curve analyses have dependencies $M_{p} \propto M_{\star}^{2 / 3}$ and $R_{p} \propto R_{\star}$, the above errors can translate into biases (overestimates) of $13 \%$ and $10 \%$ in the planetary masses and radii. For cooler stars the errors tend to be in the opposite direction, and are smaller. Analogous results were obtained when using the determinations from SPC. In the case of MOOG, on the other hand, the effect on $M_{\star}$ and $R_{\star}$ from using unconstrained temperatures and metallicities is only marginally significant, consistent with the much smaller correlations seen in Figure 4 .

We defer a comprehensive examination and tabulation of stellar masses and radii for a future paper, since we are also compiling many new light curves for analysis, which may improve the external constraints.

\section{DISCUSSION}

While most transiting planet investigations use stellar evolution models to infer stellar masses and radii, others use empirical relations for $M_{\star}$ and $R_{\star}$ as a function of temperature, metallicity, and mean density. The biases described above can affect both. The Kepler Mission is increasingly making use of asteroseismology as an alternate way of deriving the mean stellar density, based on oscillation frequencies measured directly from the light curves in favorable cases. Deriving the stellar mass or radius with this technique still requires stellar models, as well as accurate measurements of $T_{\text {eff }}$ and $[\mathrm{Fe} / \mathrm{H}]$, so there is still a danger of systematic errors from the use of unconstrained spectroscopic determinations.

Numerous investigations have addressed the persistent problem of the anomalously "inflated" radii of some of the Jovian planets - objects that can exceed $60 \%$ of the size of Jupiter in extreme cases which has been with us since the discovery of tran- 
sits in HD 209458, challenging our understanding of planet formation and evolution. A variety of mechanisms have been proposed that may play a role in "puffing up" the planets, but no universal process seems to account for all of these anomalies (see, e.g., Burrows et al. 2007; Miller et al. 2009; Fortney \& Nettelmann 2010; Demory \& Seager 2011, and references therein). Our results in the preceding section suggest that a portion of the discrepancy in $R_{p}$ may have to do with systematic errors in the stellar radii in some cases, which can amount to $\sim 10 \%$, as illustrated in Figure [1].

One of the notorious examples of inflated planets for which we have derived new spectroscopic parameters is WASP-12 b, with a radius of $R_{p}=1.79 \pm 0.09 R_{\text {Jup }}$ (Hebb et al. 2009). ${ }^{11}$ This study of WASP-12 b adopted spectroscopic properties derived from an unconstrained SME analysis yielding $T_{\text {eff }}=6290 \mathrm{~K}$ (rounded off by the authors to $6300 \mathrm{~K}$, and assigned errors of ${ }_{-100}^{+200} \mathrm{~K}$ ) and $[\mathrm{M} / \mathrm{H}]=+0.30_{-0.15}^{+0.05}$. These are both somewhat higher than we derive here from our constrained spectroscopic analyses: $T_{\text {eff }}=6118 \pm 64 \mathrm{~K}$ and $[\mathrm{Fe} / \mathrm{H}]=$ $+0.07 \pm 0.07$. We note that their spectroscopic surface gravity $(\log g=4.38)$ is also larger than the densitybased (photometrically constrained) value they reported, $\log g=4.17 \pm 0.03$. These differences are all consistent with the magnitude and sign of correlations shown in Figure 4, and leave open the possibility that the stellar mass and radius of Hebb et al. (2009) may be biased. To test this we repeated their isochrone analysis using the Yonsei-Yale stellar evolution models of Yi et al. (2001), first with the Hebb et al. (2009) temperature and metallicity, and then adopting ours. The surface gravity was held fixed at the density-based value they determined. The stellar radius we obtained with our revised spectroscopic parameters is $5 \%$ smaller, implying a planetary radius also $5 \%$ smaller, all else being equal. While this correction is far from what would be needed to solve the puzzle of the inflated radius of WASP-12 b, it does go in the right direction.

\section{CONCLUSIONS}

Accurate knowledge of the properties of the host stars in transiting exoplanet systems is essential to derive accurate characteristics for the planets. Considerable efforts have been devoted to improving the light curves of newly discovered as well as previously known transiting systems, but relatively little attention has been paid to refining the spectroscopic properties of the stars.

Here we have derived new effective temperatures, metallicities, and projected rotational velocities for 56 transiting planet systems in a homogeneous manner, using the SPC technique. These determinations bring a needed measure of uniformity to the growing collection of stellar and planetary properties that should facilitate the discovery of patterns and correlations that may provide valuable insight into the nature of planets.

A key aspect of our spectral analysis is the application of an external constraint on the surface gravity, based on

\footnotetext{
11 Chan et al. (2011) have recently revised this value downward
} slightly to $R_{p}=1.736 \pm 0.092 R_{\mathrm{Jup}}$, adopting the same spectro- accurate knowledge of the mean stellar density of the star, which comes directly from the light curve modeling. Because $\log g$ is usually weakly constrained by the spectra, fixing it as we have done here prevents errors in $\log g$ from biasing the temperatures and metallicities, and from affecting the inferred stellar masses and radii. Those biases come mainly from strong correlations between $T_{\text {eff }},[\mathrm{Fe} / \mathrm{H}]$, and $\log g$ that are present in unconstrained determinations ( $\log g$ free), not only when applying SPC, but also in the widely used SME procedure as implemented by Valenti \& Fischer (2005). Both of these methods are based on spectral synthesis. We find that the correlations are much smaller with MOOG, which uses a more classical curve-of-growth approach.

We investigate the interagreement among the three spectroscopic techniques by applying SME and MOOG to subsets of our stars, and we show that the temperatures and metallicities are generally in good accord after application of the $\log g$ constraint (the mean differences being well under $50 \mathrm{~K}$ and 0.1 dex, respectively). We do, however, detect some remaining systematic trends as a function of temperature and metallicity that are occasionally larger in some regimes.

Virtually all current studies of transiting planets make use of the mean stellar density as a luminosity indicator to derive the stellar properties, either through a comparison with model isochrones, or using empirical relations. We demonstrate that not using $\rho_{\star}$ can incur errors in mass of up to $20 \%$, and errors in the radius as large as $100 \%$ in some cases. Even though such errors are now usually avoided, many authors still retain the temperatures and metallicities obtained from unconstrained spectroscopic analyses, i.e., without fixing $\log g$ to the more accurate values based on the light curve modeling. We demonstrate that this practice can lead to residual biases in $M_{\star}$ of up to $20 \%$, and systematic errors in $R_{\star}$ up to $10 \%$ for the hotter stars, which will propagate through to the planetary properties. Such errors can be larger than other observational uncertainties, and may explain part of radius anomaly of some of the inflated Jovian planets. In order to avoid this, we advocate performing a second iteration on the spectroscopic analysis (particularly when using SPC or SME) that fixes $\log g$ to the value inferred from the photometrically determined mean stellar density.

We are grateful to Jeff Valenti for helpful discussions, and to P. Berlind, M. Calkins, G. Esquerdo, D. W. Latham, and R. P. Stefanik for their help in obtaining the TRES spectra used here. We also thank the anonymous referee for helpful comments. GT acknowledges partial support for this work from NASA's Origins of Solar Systems program, through grant NNX09AF59G, and from NSF grant AST-10-07992. The research has made use of the SIMBAD database, operated at CDS, Strasbourg, France, and of NASA's Astrophysics Data System Abstract Service.

scopic parameters as the original authors. 
Anderson, D. R. 2010a, ApJ, 709, 159

Anderson, D. R., Gillon, M., Maxted, P. F. L., et al. 2010b, A\&A, 513, L3

Anderson, D. R., Collier Cameron, A., Hellier, C., et al. 2011, A\&A, $531, \mathrm{~A} 60$

Bakos, G., Noyes, R. W., Kovács, G., Stanek, K. Z., Sasselov, D. D., \& Domsa, I. 2004, PASP, 116, 266

Bakos, G. Á., Shporer, A., Pál, A., et al. 2007, ApJ, 671, L173

Bakos, G. Á., Torres, G., Pál, A., et al. 2010, ApJ, 710, 1724

Bakos, G. Á., Howard, A. W., Noyes, R. W., et al. 2009, ApJ, 707, 446

Bakos, G. Á., Hartman, J., Torres, G., et al. 2011, ApJ, 742, 116

Barros, S. C. C., Faedi, F., Collier Cameron, A., et al. 2011, A\&A 525, A54

Batalha, N. M., Borucki, W. J., Bryson, S. T., et al. 2011, ApJ, 729,27

Bean, J. L. 2009, A\&A, 506, 369

Behr, B. B. 2003, ApJS, 149, 101

Buchhave, L. A. 2010a, Ph.D. thesis, Univ. of Copenhagen, http://intersigma.dk/data/thesis/Thesis_Lars_Buchhave.pdf

Buchhave, L. A. et al. 2010b, ApJ, 720, 1118

Buchhave, L. A. et al. 2012, Nature, 486, 375

Buchhave, L. A., Bakos, G. Á., Hartman, J. D., et al. 2011, ApJ, 733,116

Burke, C. J., McCullough, P. R., Bergeron, L. E., et al. 2010, ApJ, 719,1796

Burrows, A., Hubeny, I., Budaj, J., \& Hubbard, W. B. 2007, ApJ 661,502

Castelli, F., \& Kurucz, R. L. 2003, in Modelling of Stellar Atmospheres, IAU Symp. 210, eds. N. Piskunov, W. W. Weiss, and D. F. Gray (San Francisco: ASP), p. A20

Castelli, F., \& Kurucz, R. L. 2004, arXiv:astro-ph/0405087

Chan, T., Ingemyr, M., Winn, J. N., Holman, IV. J., Sanchis-Ojeda, R., Esquerdo, G., \& Everett, M. 2011, AJ, 141, 179

Christensen-Dalsgaard, J., Kjeldsen, H., Brown, T. M., et al. 2010, ApJ, 713, L164

Christian, D. J., Gibson, N. P., Simpson, E. K., et al. 2009, MNRAS, 392, 1585

Deleuil, M. et al. 2008, A\&A, 491, 889

Demory, B.-O., \& Seager, S. 2011, ApJS, 197, 12

Djupvic, A. A., \& Andersen, J. 2010, in Highlights of Spanish Astrophysics V, ed. J. M. Diego, L. J. Goicoechea, J. I. GonzálezSerrano, \& J. Gorgas (Berlin: Springer), 211

Enoch, B., Collier Cameron, A., Parley, N. R., \& Hebb, L. 2010, A\&A, 516, 33

Feigelson, E. D., \& Babu, G. J. 1992, ApJ, 397, 55

Fernandez, J. M., Holman, M. J., Winn, J. N., et al. 2009, AJ, 137, 4911

Fortney, J. J., \& Nettelmann, N. 2010, Space Sci. Rev., 152, 423

Fürész, G. 2008, PhD thesis, Univ. Szeged

Gilliland, R. L., McCullough, P. R., Nelan, E. P., et al. 2011, ApJ 726,2

Gillon, M., Lanotte, A. A., Barman, T., et al. 2010, A\&A, 511, A3

Girardi, L., Bressan, A., Bertelli, G., \& Chiosi, C. 2000, A\&AS, 141,371

Glazunova, L. V., Yushchenko, A. V., Tsymbal, V. V., Mkrtichian, D. E., Lee, J., J., Kang, Y. W., Valyavin, G. G., \& Lee, B.-C. 2008, AJ, 136, 1736

Hartman, J. D., Bakos, G. Á., Sato, B., et al. 2011a, ApJ, 726, 52

Hartman, J. D., Bakos, G. Á., Kipping, D. M., et al. 2011b, ApJ, 728,138

Hebb, L. et al. 2009, ApJ, 693, 1920

Hébrard, G., Désert, J.-M., Díaz, R. F., et al. 2010, A\&A, 516, A95

Hensberge, H., Pavlovski, K., \& Verschueren, W. 2000, A\&A, 358, 553

Holman, M. J. et al. 2007, ApJ, 664, 1185

Howard, J. A et al. 2012, ApJ, 749, 134

Joshi, Y. C., Pollacco, D., Collier Cameron, A., et al. 2009, MNRAS, 392, 1532

Kipping, D. M., Bakos, G. Á., Hartman, J., et al. 2010, ApJ, 725, 2017

Kipping, D., \& Bakos, G. 2011a, ApJ, 730, 50

Kipping, D., \& Bakos, G. 2011b, ApJ, 733, 36
Kovács, G. et al. 2007, ApJ, 670, L41

Kovács, G., Bakos, G. Á., Hartman, J. D., et al. 2010, ApJ, 724, 866

Kurucz, R. L. 1993, ATLAS9 Stellar Atmosphere Programs and 2 $\mathrm{km} / \mathrm{s}$ Grid CDROM 13 (Cambridge:SAO)

Latham, D. W. et al. 2002, AJ, 124, 1144

Latham, D. W., Bakos, G. Á., Torres, G., et al. 2009, ApJ, 704, 1107

Léger, A., Rouan, D., Schneider, J., et al. 2009, A\&A, 506, 287

McCullough, P. R. et al. 2006, ApJ, 648, 1228

McCullough, P. R. et al. 2008, arXiv:0805.2921

Miller,, N., Fortney, J. J., \& Jackson, B. 2009, ApJ, 702, 1413

Narita, N., Hirano, T., Sanchis-Ojeda, R., et al. 2010, PASJ, 62 , L61

Noyes, R. W., Bakos, G. Á., Torres, G., et al. 2008, ApJ, 673, L79

Pál, A., Bakos, G. Á., Torres, G., et al. 2010, MNRAS, 401, 2665

Perryman, M. A. C., et al. 1997, The Hipparcos and Tycho Catalogues (ESA SP-1200; Noordwiik: ESA)

Pollacco, D. L. et al. 2006, PASP, 118, 1407

Press, W. H., Teukolsky, S. A., Vetterling, W. T., \& Flannery, B. P. 1992, Numerical Recipes, (2nd. Ed.; Cambridge: Cambridge Univ. Press), 650

Quinn, S. N., Bakos, G. Á., Hartman, J., et al. 2012, ApJ, 745, 80

Royer, R., Grenier, S., Baylac, M.-O., Gómez, A. E., \& Zorec, J. $2002 \mathrm{~b}, \mathrm{~A} \& \mathrm{~A}, 393,897$

Royer, R., Gerbaldi, M., Faraggiana, R., \& Gómez, A. E. 2002a, A\&A, 381, 105

Santos, N. C., Israelian, G., \& Mayor, M. 2004, A\&A, 415, 1153

Santos, N. C. et al. 2006, A\&A, 450, 825

Schuler, S. C., Plunkett, A. L., King, J. R., \& Pinsonneault, M. H. 2010, PASP, 122, 766

Seager, S., \& Mallén-Ornelas, G. 2003, ApJ, 585, 1038

Shporer, A., Bakos, G. Á., Bouchy, F., et al. 2009, ApJ, 690, 1393

Simpson, E. K., Pollacco, D., Hébrard, G., et al. 2010, MNRAS, 405,1867

Skillen, I., Pollacco, D., Collier Cameron, A., et al. 2009, A\&A, 502,39

Smalley, B., Smith, K., \& Dworetsky, M. 2001, http://www.astro.keele.ac.uk/ bs/publs/uclsyn.pdf

Sneden, C. A. 1973, Ph.D. thesis, Univ. Texas (Austin)

Southworth, J. 2009a, MNRAS, 394, 272

Southworth, J., Hinse, T. C., Dominik, M., et al. 2009b, ApJ, 707, 167

Sousa, S. G., Santos, N. C., Israelian, G., Mayor, M., \& Monteiro, M. J. P. F. G. 2007, A\&A, 469, ,783

Sozzetti, A., Yong, D., Carney, B. W., Laird, J. B., Latham, D.

W., \& Torres, G. 2006, AJ, 131, 2274

Sozzetti, A., Yong, D., Torres, G., Charbonneau, D., Latham, D.

W., Holman, M. J., Winn, J. N., Laird, J. B., \& O'Donovan, F. T. 2007, ApJ, 664, 1190

Sozzetti, A. et al. 2009, ApJ, 691, 1145

Street, R. A., Simpson, E., Barros, S. C. C., et al. 2010, ApJ, 720, 337

Torres, G., Neuhäuser, R., \& Guenther, E. W. 2002, AJ, 123, 1701

Torres, G., Bakos, G. Á., Kovács, G., et al. 2007, ApJ, 666, L121

Torres, G., Winn, J. N., \& Holman, M. J. 2008, ApJ, 677, 1324

Torres, G., Bakos, G. Á., Hartman, J., et al. 2010b, ApJ, 715, 458

Torres, G., Andersen, A., \& Giménez, A. 2010a, A\&ARv, 18, 67

Valenti, J. A., \& Fischer, D. A. 2005, ApJS, 159, 142

Valenti, J. A., \& Piskunov, N. 1996, A\&AS, 118, 595

van Leeuwen, F. 2007, Hipparcos, the New Reduction of the Raw

Data, Astr. and Sp. Sci. Library, Vol. 350 (Cambridge: Springer)

Vogt, S. S. et al. 1994, Proc. SPIE, 2198, 362

West, R. G., Collier Cameron, A., Hebb, L., et al. 2009, A\&A, 502, 395

Winn, J. N., Holman, M. J., Torres, G., et al. 2008, ApJ, 683, 1076

Yi, S. K., Demarque, P., Kim, Y.-C., Lee, Y.-W., Ree, C. H., Lejeune, T., \& Barnes, S. 2001, ApJS, 136, 417

Yong, D., Lambert, D. L., Allende Prieto, C., \& Paulson, D. B. 2004, ApJ, 603, 697 